\title{
A Two-Mode Mean-Field Optimal Switching Problem for the Full Balance Sheet
}

\author{
Boualem Djehiche and Ali Hamdi \\ Department of Mathematics, KTH-Royal Institute of Technology, 10044 Stockholm, Sweden \\ Correspondence should be addressed to Boualem Djehiche; boualem@math.kth.se
}

Received 20 February 2014; Accepted 4 May 2014; Published 25 May 2014

Academic Editor: Qing Zhang

Copyright (C) 2014 B. Djehiche and A. Hamdi. This is an open access article distributed under the Creative Commons Attribution License, which permits unrestricted use, distribution, and reproduction in any medium, provided the original work is properly cited.

\begin{abstract}
We consider the problem of switching a large number of production lines between two modes, high production and low production. The switching is based on the optimal expected profit and cost yields of the respective production lines and considers both sides of the balance sheet. Furthermore, the production lines are all assumed to be interconnected through a coupling term, which is the average of all optimal expected yields. Intuitively, this means that each individual production line is compared to the average of all its peers which acts as a benchmark. Due to the complexity of the problem, we consider the aggregated optimal expected yields, where the coupling term is approximated with the mean of the optimal expected yields. This turns the problem into a twomode optimal switching problem of mean-field type, which can be described by a system of Snell envelopes where the obstacles are interconnected and nonlinear. The main result of the paper is a proof of a continuous minimal solution to the system of Snell envelopes, as well as the full characterization of the optimal switching strategy.
\end{abstract}

\section{Introduction}

Consider a company with $N$ different production lines which all have two modes of production, high mode and low mode, where each mode of production has its own balance sheet of expected profits and costs. For each production line $j$ in mode $i$, let $Y_{t}^{+, i, j}$ denote the optimal expected profit yield at time $t$ and let the corresponding optimal expected cost yield be denoted by $Y_{t}^{-, i, j}$. Assume that we want to switch between the two modes of production either if the current mode is unprofitable or if we can expect better profits in the other mode. Assume further that the switching is based on both sides of the balance sheet, so that we, for example, switch if we can expect lower costs in the other mode. Then this problem can be modeled as a two-mode optimal switching problem for each production line $j$, which can be described by the following system of Snell envelopes:

$$
\begin{aligned}
& Y_{t}^{+, i, j} \\
& \quad=\underset{\tau \geq t}{\operatorname{ess} \sup } \mathbb{E}\left[\int_{t}^{\tau} \psi_{i}^{+, j}(s) d s+S_{\tau}^{+, i, j} \mathbf{1}_{\{\tau<T\}}+\xi_{i}^{+} \mathbf{1}_{\{\tau=T\}} \mid \mathscr{F}_{t}\right],
\end{aligned}
$$

$$
\begin{aligned}
& Y_{t}^{-, i, j} \\
& \quad=\underset{\tau \geq t}{\operatorname{essinf}} \mathbb{E}\left[\int_{t}^{\tau} \psi_{i}^{-, j}(s) d s+S_{\tau}^{-, i, j} \mathbf{1}_{\{\tau<T\}}+\xi_{i}^{-} \mathbf{1}_{\{\tau=T\}} \mid \mathscr{F}_{t}\right],
\end{aligned}
$$

for each production line $j=1, \ldots, N$, where $\psi_{i}^{ \pm j}(s)$ are the profit and cost rates per unit time (the generators) and where

$$
\begin{aligned}
& S_{t}^{+, 1, j}=\left(Y_{t}^{+, 2, j}-\ell_{1}(t)\right) \vee\left(Y_{t}^{-, 1, j}-a_{1}(t)\right), \\
& S_{t}^{+, 2, j}=\left(Y_{t}^{+, 1, j}-\ell_{2}(t)\right) \vee\left(Y_{t}^{-, 2, j}-a_{2}(t)\right), \\
& S_{t}^{-, 1, j}=\left(Y_{t}^{-, 2, j}+\ell_{1}(t)\right) \wedge\left(Y_{t}^{+, 1, j}+b_{1}(t)\right), \\
& S_{t}^{-, 2, j}=\left(Y_{t}^{-, 1, j}+\ell_{2}(t)\right) \wedge\left(Y_{t}^{+, 2, j}+b_{2}(t)\right)
\end{aligned}
$$

are the obstacles of the switching problem. Here, $\xi_{i}^{ \pm}$are the final profits and costs of each mode $i$ at some fixed time $T$ and the functions $\ell_{i}, a_{i}$, and $b_{i}$ represent switching costs. 
Now assume that the production lines all have the same generators but they are interconnected through a coupling term. If the coupling term is the average of the profit and cost yields of all the projects,

$$
\frac{1}{N} \sum_{j=1}^{N} Y_{t}^{ \pm, i, j}
$$

then this intuitively means that each production line is compared to a benchmark constituted of the average of its peers. In this case, the corresponding generators become

$$
\begin{aligned}
& \psi_{i}^{+, j}(t)=\psi_{i}^{+}\left(t, Y_{t}^{+, i, j}, \frac{1}{N} \sum_{k=1}^{N} Y_{t}^{+, i, k}\right), \\
& \psi_{i}^{-, j}(t)=\psi_{i}^{-}\left(t, Y_{t}^{-, i, j}, \frac{1}{N} \sum_{k=1}^{N} Y_{t}^{-, i, k}\right) .
\end{aligned}
$$

With this assumption, solving the system (1) becomes a highly complex task for large $N$, since the Snell envelopes are all interconnected through the coupling term (3). But instead of solving (1) we can consider the expected profit and cost yields on an aggregated level, where we use the mean-field approximation $\mathbb{E} Y_{t}^{ \pm, i}$ for the coupling term (3). The corresponding system of Snell envelopes becomes

$$
\begin{aligned}
& Y_{t}^{+, i}=\underset{\tau \geq t}{\operatorname{ess} \sup } \mathbb{E} {\left[\int_{t}^{\tau} \psi_{i}^{+}\left(s, Y_{s}^{+, i}, \mathbb{E} Y_{s}^{+, i}\right) d s\right.} \\
&\left.+S_{s}^{+, i} \mathbf{1}_{\{\tau<T\}}+\xi_{i}^{+} \mathbf{1}_{\{\tau=T\}} \mid \mathscr{F}_{t}\right], \\
& Y_{t}^{-, i}=\underset{\tau \geq t}{\operatorname{ess} \inf _{t} \mathbb{E}[}\left[\int_{t}^{\tau} \psi_{i}^{+}\left(s, Y_{s}^{-, i}, \mathbb{E} Y_{s}^{-, i}\right) d s\right. \\
&\left.+S_{s}^{-, i} \mathbf{1}_{\{\tau<T\}}+\xi_{i}^{-} \mathbf{1}_{\{\tau=T\}} \mid \mathscr{F}_{t}\right],
\end{aligned}
$$

for $i=1,2$, where

$$
\begin{aligned}
& S_{t}^{+, 1}=\left(Y_{t}^{+, 2}-\ell_{1}(t)\right) \vee\left(Y_{t}^{-, 1}-a_{1}(t)\right), \\
& S_{t}^{+, 2}=\left(Y_{t}^{+, 1}-\ell_{2}(t)\right) \vee\left(Y_{t}^{-, 2}-a_{2}(t)\right), \\
& S_{t}^{-, 1}=\left(Y_{t}^{-, 2}+\ell_{1}(t)\right) \wedge\left(Y_{t}^{+, 1}+b_{1}(t)\right), \\
& S_{t}^{-, 2}=\left(Y_{t}^{-, 1}+\ell_{2}(t)\right) \wedge\left(Y_{t}^{+, 2}+b_{2}(t)\right) .
\end{aligned}
$$

In this paper we will show the existence of a continuous minimal solution of this system. In a forthcoming paper we will show convergence of the system (1) to our system of Snell envelops of mean-field type. The set of counterexamples derived in [1] can be used to argue that uniqueness may not hold in general.
In terms of BSDEs, the system is equivalent to the following system of mean-field reflected BSDEs (MF-RBSDEs):

$$
\begin{gathered}
Y_{t}^{+, i}=\xi_{i}^{+}+\int_{t}^{\tau} \psi_{i}^{+}\left(s, Y_{s}^{+, i}, \mathbb{E} Y_{s}^{+, i}\right) d s+\left(K_{T}^{+, i}-K_{t}^{+, i}\right) \\
-\int_{t}^{T} Z_{s}^{+, i} d B_{s}, \\
Y_{t}^{-, i}=\xi_{i}^{-}+\int_{t}^{\tau} \psi_{i}^{-}\left(s, Y_{s}^{-, i}, \mathbb{E} Y_{s}^{-, i}\right) d s-\left(K_{t}^{-, i}-K_{t}^{-, i}\right) \\
-\int_{t}^{T} Z_{s}^{-, i} d B_{s}, \\
Y_{t}^{+, i} \geq S_{t}^{+, i}, \quad Y_{t}^{-, i} \leq S_{t}^{-, i}, \\
\quad \int_{0}^{T}\left(Y_{t}^{+, i}-S_{t}^{+, i}\right) d K_{t}^{+, i}=0, \\
\int_{0}^{T}\left(S_{t}^{-, i}-Y_{t}^{-, i}\right) d K_{t}^{-, i}=0 .
\end{gathered}
$$

For details, see, for example, [2].

In this paper we consider the following slightly more general system of MF-RBSDEs:

$$
\begin{gathered}
Y_{t}^{+, i}=\xi_{i}^{+}+\int_{t}^{\tau} \psi_{i}^{+}\left(s, Y_{s}^{+, i}, \mathbb{E} Y_{s}^{+, i}, Z_{s}^{+, i}\right) d s+\left(K_{T}^{+, i}-K_{t}^{+, i}\right) \\
-\int_{t}^{T} Z_{s}^{+, i} d B_{s}, \\
Y_{t}^{-, i}=\xi_{i}^{-}+\int_{t}^{\tau} \psi_{i}^{-}\left(s, Y_{s}^{-, i}, \mathbb{E} Y_{s}^{-, i}, Z_{s}^{-, i}\right) d s-\left(K_{t}^{-, i}-K_{t}^{-, i}\right) \\
-\int_{t}^{T} Z_{s}^{-, i} d B_{s}, \\
Y_{t}^{+, i} \geq S_{t}^{+, i}, \quad Y_{t}^{-, i} \leq S_{t}^{-, i}, \\
\int_{0}^{T}\left(Y_{t}^{+, i}-S_{t}^{+, i}\right) d K_{t}^{+, i}=0, \\
\int_{0}^{T}\left(S_{t}^{-, i}-Y_{t}^{-, i}\right) d K_{t}^{-, i}=0 .
\end{gathered}
$$

We follow a procedure similar to the one used in [1]; that is, we use an increasing sequence of approximating meanfield reflected stochastic differential equations (MF-RBSDEs) to show the existence of a continuous minimal solution of the system. However, due to the added generality in the problem, we have to prove a comparison result and an upper bound for this type of MF-RBSDE given the in-data.

Mean-field related problems have been studied not only in the setting of backward stochastic differential equations, but in many other fields as well. Examples of areas where mean-field approximations have been successful include statistical mechanics, quantum mechanics, quantum chemistry, economics, finance, and game theory. Recent work includes, for example, [3], where the authors consider the problem 
of sector-wise allocation in a portfolio consisting of a very large number of stocks. Another paper on mean-field approximation is the seminal work by Lasry and Lions [4], which concerns applications of mean-field approximations to problems in economics and finance. For an account on recent work related to our paper we refer to [5] and the references therein.

Backward stochastic differential equations of the meanfield type have been studied by several authors including [58]. To the best of our knowledge, the work of Buckdahn et al. [6] is the first paper to tackle this class of problems. They study an equation of the form

$$
\begin{aligned}
Y_{t}= & \xi+\int_{t}^{T} \mathbb{E}^{\prime} f\left(s, \omega^{\prime}, \omega, Y_{s}\left(\omega^{\prime}\right), Z_{s}\left(\omega^{\prime}\right), Y_{s}(\omega), Z_{s}(\omega)\right) d s \\
& -\int_{t}^{T} Z_{s} d W_{s},
\end{aligned}
$$

where the mean-field interaction is linear in the generator, obtained as a mean-field limit of BSDE equation driven by SDEs of mean-field type. This type of mean-field backward stochastic differential equation (MF-BSDE) is further studied in [6] where the authors obtain existence and uniqueness for a general driver, under Lipschitz conditions. Extensions of this work to reflected BSDEs include $[7,8]$. The equations they study are of the form

$$
\begin{gathered}
Y_{t}=\xi+\int_{t}^{T} \mathbb{E}^{\prime} f\left(s, \omega^{\prime}, \omega, Y_{s}\left(\omega^{\prime}\right),\right. \\
\left.Z_{s}\left(\omega^{\prime}\right), Y_{s}(\omega), Z_{s}(\omega)\right) d s \\
+K_{T}-K_{t}-\int_{t}^{T} Z_{s} d W_{s}, \\
Y_{t} \geq S_{t}, \quad \int_{0}^{T}\left(Y_{t}-S_{t}\right) d K_{t}=0 .
\end{gathered}
$$

The authors prove existence and uniqueness of the MFBSDEs as well as a comparison theorem under some additional conditions for the generator. These results easily extend to our case where the mean-field interaction of the MFRBSDEs is nonlinear in the generator. For completeness, we display in the Appendices an adaptation of the proof of $[7,8]$ to our setting.

The outline of this paper is as follows. Section 2 states the necessary notation and preliminaries. In Section 3 we state and prove the main results of this paper.

\section{Notation and Preliminaries}

For the rest of the paper we fix a probability space, denoted by $(\Omega, \mathscr{F}, \mathbb{P})$, on which is defined as a standard $d$-dimensional Brownian motion $B=\left(B_{t}\right)_{0 \leq t \leq T}$ whose natural filtration is $\left(\mathscr{F}_{t}^{0}:=\sigma\left\{B_{s}, s \leq t\right\}\right)_{0 \leq t \leq T}$. Let $\mathbb{F}=\left(\mathscr{F}_{t}\right)_{0 \leq t \leq T}$ be the filtration $\left(\mathscr{F}_{t}^{0}\right)_{0 \leq t \leq T}$ completed with the $\mathbb{P}$-null sets of $\mathscr{F}$. This implies that $\mathbb{F}$ satisfies the usual conditions; that is, it is right continuous and complete.

For future reference, we introduce the following spaces:

(i) $\mathscr{P}$ is the $\sigma$-algebra on $[0, T] \times \Omega$ of $\mathbb{F}$-progressively measurable processes,

(ii) $\mathscr{M}^{d, 2}$ is the set of $\mathscr{P}$-measurable and $\mathbb{R}^{d}$-valued processes $w=\left(w_{t}\right)_{0 \leq t \leq T}$ such that

$$
\|w\|_{\mathscr{M}^{d, 2}}:=\mathbb{E}\left[\int_{0}^{T}\left|w_{s}\right|^{2} d s\right]^{1 / 2}<\infty
$$

(iii) $\mathcal{S}^{2}$ (resp., $\mathcal{S}_{c}^{2}$ ) is the set of $\mathscr{P}$-measurable and càdlàg (resp., continuous) $\mathbb{R}$-valued processes $w=\left(w_{t}\right)_{0 \leq t \leq T}$ such that

$$
\|w\|_{S^{2}}:=\mathbb{E}\left[\sup _{0 \leq t \leq T}\left|w_{t}\right|^{2}\right]^{1 / 2}<\infty
$$

(iv) $\mathscr{K}^{2}$ (resp., $\mathscr{K}_{c}^{2}$ ) is a subset of $\mathcal{S}^{2}$ (resp., $\mathcal{S}_{c}^{2}$ ) on nondecreasing càdlàg (resp., continuous) processes $\left(K_{t}\right)_{0 \leq t \leq T}$ such that $K_{0}=0$.

Let $\xi$ be an $\mathscr{F}_{T}$-measurable $L^{2}$-random variable, let $f$ be an $\mathbb{R}$-valued function, and let $S$ be an $\mathbb{F}$-adapted process. To streamline the presentation of the results, we introduce the following notation.

(i) If there exist a pair of processes $(Y, Z)$ such that

$$
\begin{gathered}
Y \in \mathcal{S}^{2}\left(\text { resp., } \mathcal{S}_{c}^{2}\right), \quad Z \in \mathscr{M}^{d, 2}, \\
Y_{t}=\xi+\int_{t}^{T} f\left(s, \omega, Y_{s}, \mathbb{E} Y_{s}, Z_{s}\right) d s-\int_{t}^{T} Z_{s} d B_{s}, \\
\text { then we say that }
\end{gathered}
$$

$$
(Y, Z)=F(\xi, f) \quad\left(\text { resp., }(Y, Z)=F^{c}(\xi, f)\right) .
$$

(ii) If there exist a triple of processes $(Y, Z, K)$ such that

$$
\begin{gathered}
\left.Y \in \mathcal{S}^{2}\left(\text { resp., } \mathcal{S}_{c}^{2}\right), \quad Z \in \mathscr{M}^{d, 2}, \quad K \in \mathscr{K}^{2} \quad \text { (resp., } \mathscr{K}_{c}^{2}\right) \\
Y_{t}=\xi+\int_{t}^{T} f\left(s, \omega, Y_{s}, \mathbb{E} Y_{s}, Z_{s}\right) d s+K_{T}-K_{t}-\int_{t}^{T} Z_{s} d B_{s}, \\
Y_{t} \geq S_{t}, \quad 0 \leq t \leq T, \\
\int_{0}^{T}\left(Y_{t^{-}}-S_{t^{-}}\right) d K_{t}=0 \quad\left(\text { resp., } \int_{0}^{T}\left(Y_{t}-S_{t}\right) d K_{t}=0\right),
\end{gathered}
$$

then we say that

$$
(Y, Z, K)=F_{+}(\xi, f, S) \quad\left(\text { resp., }(Y, Z, K)=F_{+}^{c}(\xi, f, S)\right) .
$$


(iii) If there exist a triple of processes $(Y, Z, K)$ such that

$$
\begin{gathered}
Y \in \mathcal{S}^{2}\left(\text { resp., } \mathcal{S}_{c}^{2}\right), \quad Z \in \mathscr{M}^{d, 2}, \quad K \in \mathscr{K}^{2}\left(\text { resp., } \mathscr{K}_{c}^{2}\right) \\
Y_{t}=\xi+\int_{t}^{T} f\left(s, \omega, Y_{s}, \mathbb{E} Y_{s}, Z_{s}\right) d s-\left(K_{T}-K_{t}\right)-\int_{t}^{T} Z_{s} d B_{s}, \\
Y_{t} \leq S_{t}, \quad 0 \leq t \leq T, \\
\int_{0}^{T}\left(S_{t^{-}}-Y_{t^{-}}\right) d K_{t}=0 \quad\left(\text { resp., } \int_{0}^{T}\left(Y_{t}-S_{t}\right) d K_{t}=0\right),
\end{gathered}
$$

then we say that

$$
(Y, Z, K)=F_{-}(\xi, f, S) \quad\left(\text { resp., }(Y, Z, K)=F_{-}^{c}(\xi, f, S)\right) .
$$

These mean-field backward stochastic differential equations (MF-BSDEs) and mean-field reflected backward stochastic differential equations (MF-RBSDEs) are said to be standard if the following conditions hold.

(H1) The generator $f$ is Lipschitz with respect to $\left(y_{1}, y_{2}, z\right)$ uniformly in $(t, \omega)$.

(H2) The process $(f(t, \omega, 0,0,0))_{0 \leq t \leq T}$ is $\mathbb{F}$-progressively measurable and $d t \otimes d \mathbb{P}$-square integrable.

(H3) The random variable $\xi$ is in $L^{2}\left(\Omega, \mathscr{F}_{T}, \mathbb{P}\right)$.

(H4) The barrier $S$ is càdlàg, $\mathbb{F}$-adapted and satisfies

$$
\mathbb{E}\left[\sup _{0 \leq s \leq T}\left|S_{s}^{+}\right|^{2}\right]<\infty
$$

and $S_{T} \leq \xi$, $\mathbb{P}$-a.s.

More on MF-BSDEs can be found in $[5,6]$. For further reference on MF-RBSDEs, see [7, 8].

Finally, a key tool used in this paper is the notion of the Snell envelope. Let $\mathscr{T}_{\theta}$ denote the class of $\mathbb{F}$-stopping times $\tau$ such that $\tau \geq \theta$ for some $\mathbb{F}$-stopping time $\theta$.

Proposition 1. Let $U=\left(U_{t}\right)_{0 \leq t \leq T}$ be an $\mathbb{F}$-adapted $\mathbb{R}$-valued cadlàg process such that the set of random variables $\left\{U_{\tau}, \tau \in\right.$ $\left.\mathscr{T}_{0}\right\}$ is uniformly integrable. Then there exists an $\mathbb{F}$-adapted $\mathbb{R}$ valued càdlàg process $Z:=\left(Z_{t}\right)_{0 \leq t \leq T}$ such that $Z$ is the smallest supermartingale which dominates $U$. The process $Z$ is called the Snell envelope of $U$ and it has the following properties.

(i) For any $\mathbb{F}$-stopping time $\theta$ it holds that

$$
Z_{\theta}=\underset{\tau \in \mathscr{T}_{\theta}}{\operatorname{ess} \sup } \mathbb{E}\left[U_{\tau} \mid \mathscr{F}_{\theta}\right] \quad\left(\text { and hence } Z_{T}=U_{T}\right) \text {. }
$$

(ii) The Doob-Meyer decomposition of $Z$ implies the existence of a continuous martingale $\left(M_{t}\right)_{0 \leq t \leq T}$ and two nondecreasing predictable processes $\left(A_{t}\right)_{0 \leq t \leq T}$ and $\left(B_{t}\right)_{0 \leq t \leq T}$ which are, respectively, continuous and purely discontinuous such that for all $0 \leq t \leq T$ one has

$$
Z_{t}=M_{t}-A_{t}-B_{t} \quad\left(\text { with } A_{0}=B_{0}=0\right) .
$$

(iii) For any $0 \leq t \leq T$,

$$
\left\{\Delta B_{t}>0\right\} \subseteq\left\{\Delta U_{t}<0\right\} \cap\left\{Z_{t^{-}}=U_{t^{-}}\right\} .
$$

Hence, if $U$ only has positive jumps, then $Z$ is a continuous process.

(iv) If $\theta$ is an $\mathbb{F}$-stopping time, then

$$
\tau_{\theta}^{*}:=\inf \left\{t \geq \theta: Z_{t}=U_{s}\right\} \wedge T
$$

is optimal after $\theta$; that is,

$$
\begin{aligned}
Z_{\theta} & =\underset{\tau \in \mathscr{T}_{\theta}}{\operatorname{ess} \sup } \mathbb{E}\left[U_{\tau} \mid \mathscr{F}_{\theta}\right] \\
& =\mathbb{E}\left[U_{\tau_{\theta}^{*}} \mid \mathscr{F}_{\theta}\right]=\mathbb{E}\left[Z_{\tau_{\theta}^{*}} \mid \mathscr{F}_{\theta}\right] .
\end{aligned}
$$

For further reference on the Snell envelope we refer to [9, 10] or [11].

We finally collect results regarding existence, uniqueness, bounds, and comparison for MF-RBSDEs. These are adaptations of results in $[7,8]$ to our case. Proofs are deferred to the Appendices.

Proposition 2. Let $(\xi, f, S)$ be some in-data which satisfies (H1)-(H4). Then there exists a unique triplet $(Y, Z, K)$ which solves

$$
(Y, Z, K)=F_{+}(\xi, f, S) .
$$

A similar result holds for $(Y, Z, K)=F_{-}(\xi, f, S)$.

Proof. See Appendix A.

Proposition 3. Let $(\xi, f, S)$ be a set of data satisfying assumptions $(\mathbf{H 1})-(\mathbf{H} 4)$ and let $(Y, Z, K)=F_{ \pm}(\xi, f, S)$. Then there exists a constant $C$ such that

$$
\begin{aligned}
& \mathbb{E}\left[\sup _{0 \leq t \leq T}\left|Y_{t}\right|^{2}+\int_{0}^{T}\left|Z_{t}\right|^{2} d t+\left|K_{T}\right|^{2}\right] \\
& \leq C \mathbb{E}\left[\xi^{2}+\int_{0}^{T}|f(t, 0,0,0)| d t+\sup _{0 \leq t \leq T}\left(S_{t}^{+}\right)\right] .
\end{aligned}
$$

Proof. See Appendix B.

Next, we display a comparison result for solutions of $(Y, Z, K)=F_{+}(\xi, f, S)$. A similar result holds for $(Y, Z, K)=$ $F_{-}(\xi, f, S)$.

Proposition 4. Let $(\xi, f, S)$ and $\left(\xi^{\prime}, f^{\prime}, S^{\prime}\right)$ be two sets of data, each one satisfying assumptions (H1)-(H4) and let

$$
\begin{aligned}
(Y, Z, K) & =F_{+}(\xi, f, S), \\
\left(Y^{\prime}, Z^{\prime}, K^{\prime}\right) & =F_{+}\left(\xi^{\prime}, f^{\prime}, S^{\prime}\right) .
\end{aligned}
$$

If the following conditions hold:

(i) $\xi \leq \xi^{\prime}$, a.s.,

(ii) $f\left(t, y_{1}, y_{2}, z\right) \leq f^{\prime}\left(t, y_{1}, y_{2}, z\right), d \mathbb{P} \times d t$-a.e., and $\forall\left(y_{1}, y_{2}, z\right) \in \mathbb{R} \times \mathbb{R} \times \mathbb{R}^{d}$, 
(iii) $S_{t} \leq S_{t}^{\prime}, 0 \leq t \leq T$, a.s.,

(iv) $S$ and $S^{\prime}$ are continuous,

(v) at least one of the two generators $f$ and $f^{\prime}$ is nondecreasing in $y_{2}$,

then

$$
Y_{t} \leq Y_{t}^{\prime}, \quad 0 \leq t \leq T,
$$

P-a.s.

Proof. See Appendix C.

Proposition 5. Proposition 4 holds true even when $S=S^{\prime}$ a.s. and $S$ only satisfies (H4); that is, it need not be continuous.

Proof. See Appendix D.

\section{The System of MF-RBSDEs}

Consider the following system of equations:

$$
\begin{aligned}
& \left(Y^{+, i}, Z^{+, i}, K^{+, i}\right)=F_{+}\left(\xi_{i}^{+}, \psi_{i}^{+}, S^{+, i}\right), \\
& \left(Y^{-, i}, Z^{-, i}, K^{-, i}\right)=F_{-}\left(\xi_{i}^{-}, \psi_{i}^{-}, S^{-, i}\right),
\end{aligned}
$$

for $i=1,2$, where

$$
\begin{aligned}
& S_{t}^{+, 1}=\left(Y_{t}^{+, 2}-\ell_{1}(t)\right) \vee\left(Y_{t}^{-, 1}-a_{1}(t)\right), \\
& S_{t}^{+, 2}=\left(Y_{t}^{+, 1}-\ell_{2}(t)\right) \vee\left(Y_{t}^{-, 2}-a_{2}(t)\right), \\
& S_{t}^{-, 1}=\left(Y_{t}^{-, 2}+\ell_{1}(t)\right) \wedge\left(Y_{t}^{+, 1}+b_{1}(t)\right), \\
& S_{t}^{-, 2}=\left(Y_{t}^{-, 1}+\ell_{2}(t)\right) \wedge\left(Y_{t}^{+, 2}+b_{2}(t)\right) .
\end{aligned}
$$

Further assume the following.

(A1) $\psi_{i}^{ \pm}$are Lipschitz in $\left(y_{1}, y_{2}, z\right)$ uniformly in $(t, \omega)$; that is, there exists a $C>0$ such that, for any $(t, \omega) \in$ $[0, T] \times \Omega$,

$$
\begin{aligned}
& \left|\psi_{i}^{ \pm}\left(t, \omega, y_{1}, y_{2}, z\right)-\psi_{i}^{ \pm}\left(t, \omega, y_{1}^{\prime}, y_{2}^{\prime}, z\right)\right| \\
& \quad \leq C\left(\left|y-y^{\prime}\right|+\left|z-z^{\prime}\right|\right) .
\end{aligned}
$$

In addition, the processes $\psi_{i}^{ \pm, 0}(t):=\psi_{i}^{ \pm}(t, \omega, 0,0)$ are $\mathscr{F}$-progressively measurable and $d t \otimes d \mathbb{P}$-square integrable.

(A2) The processes $\left(a_{i}(t, \omega)\right)_{0 \leq t \leq T}, \quad\left(b_{i}(t, \omega)\right)_{0 \leq t \leq T}$, and $\left(\ell_{i}(t, \omega)\right)_{0 \leq t \leq T}$ belong to $\mathcal{S}_{c}^{2}$. In addition, $\ell_{i}(t)>0 \mathbb{P}$ a.s.

(A3) The random variables $\xi_{i}^{ \pm}$are $\mathscr{F}_{T}$-measurable and square integrable. Furthermore, $\mathbb{P}$-a.s. it holds that

$$
\begin{aligned}
& \xi_{1}^{+} \geq\left(\xi_{2}^{+}-\ell_{1}(T)\right) \vee\left(\xi_{1}^{-}-a_{1}(T)\right), \\
& \xi_{2}^{+} \geq\left(\xi_{1}^{+}-\ell_{2}(T)\right) \vee\left(\xi_{2}^{-}-a_{2}(T)\right), \\
& \xi_{1}^{-} \leq\left(\xi_{2}^{-}+\ell_{1}(T)\right) \wedge\left(\xi_{1}^{+}+b_{1}(T)\right), \\
& \xi_{2}^{-} \leq\left(\xi_{1}^{-}+\ell_{2}(T)\right) \wedge\left(\xi_{2}^{+}+b_{2}(T)\right) .
\end{aligned}
$$

(A4) The processes $\left(b_{i}(t)\right)_{0 \leq t \leq T}$ and $\left(\ell_{i}(t)\right)_{0 \leq t \leq T}$ are of Itôtype; that is,

$$
\begin{gathered}
b_{i}(t)=b_{i}(0)+\int_{0}^{t} U_{i}(s) d s+\int_{0}^{t} V_{i}(s) d B_{s}, \\
\ell_{i}(t)=\ell_{i}(0)+\int_{0}^{t} \bar{U}_{i} d s+\int_{0}^{t} \bar{V}_{i} d B_{s}
\end{gathered}
$$

where $(U, \bar{U})$ and $(V, \bar{V})$ are some $\mathbb{F}$-progressively measurable processes which are $d t \otimes \mathbb{P}$-square integrable.

It is worth noting a few things here. First, the obstacle processes $a_{i}$ need not be of Itô-type. Second, the set of solutions to the system (32) is nonempty. An example of solution is to set $\xi_{i}^{+}=\xi_{i}^{-}=1$, and let

$$
\begin{gathered}
\ell_{i}(T)=e^{-4 T}, \\
a_{i}(T)=b_{i}(T)=0,
\end{gathered}
$$

for $i=1,2$. Lastly, while $Y^{+, i}$ is a supermartingale, $Y^{-, i}$ is a submartingale. The assumption (A4) is needed to prove the continuity of the increasing process $K^{-, i}$. This is in turn used to prove continuity of $Y^{-, i}$, which finally is used to derive continuity of $Y^{+, i}$.

Theorem 6. Let the generators in the system (29) be nondecreasing in the third argument; that is,

$$
y_{2} \longrightarrow \psi_{i}^{ \pm}\left(t, y_{1}, y_{2}, z\right), \quad i=1,2 \text {, }
$$

are nondecreasing functions. Then, under the assumptions (A1)-(A4), the system (29) admits a minimal solution such that $\rightarrow Y_{t}^{ \pm, i}, i=1,2$, is continuous. The solution is minimal in the sense that if $\left(\widetilde{Y}^{ \pm, i}, \widetilde{Z}^{ \pm, i}, \widetilde{K}^{ \pm, i}\right), i=1,2$, is another solution, then $Y_{t}^{ \pm, i} \leq \tilde{Y}_{t}^{ \pm, i}, 0 \leq t \leq T$, a.s. The solution to the system (32) is not unique in general.

Proof. The theorem is proved using an approximating scheme. Let

$$
\left(Y^{+, i, 0}, Z^{+, i, 0}\right)=F\left(\xi_{i}^{+}, \psi_{i}^{+}\right)
$$

and denote

$$
L_{t}^{i}:=Y_{t}^{+, i, 0}+b_{i}(t)
$$

Then

$$
\begin{aligned}
L_{t}^{i}= & \xi_{i}^{+}+\int_{t}^{T} \psi_{i}^{+}\left(s, Y_{s}^{+, i, 0}, \mathbb{E} Y_{s}^{+, i, 0}, Z_{s}^{+, i, 0}\right) d s-\int_{t}^{T} Z_{s}^{+, i, 0} d B_{s} \\
& +b_{i}(T)-\int_{t}^{T} U_{i}(s) d s-\int_{t}^{T} V_{i}(s) d B_{s} \\
= & L_{T}^{i}+\int_{t}^{T} \psi_{i}\left(s, L_{s}^{i}, \mathbb{E} L_{s}^{i}, Z_{s}^{i}\right) d s-\int_{t}^{T} Z_{s}^{i} d B_{s},
\end{aligned}
$$


where

$$
\begin{aligned}
& Z_{t}^{i}:=Z_{t}^{+, i, 0}+V_{i}(t), \\
& \psi_{i}\left(s, L_{s}^{i}, \mathbb{E} L_{s}^{i}, Z_{s}^{i}\right) \\
& \quad:=\psi_{i}^{+}\left(s, L_{s}^{i}-b_{i}(s), \mathbb{E} L_{s}^{i}-\mathbb{E} b_{i}(s), Z_{s}^{i}-V_{i}(s)\right)-U_{i}(s) .
\end{aligned}
$$

Now let $(\dot{Y}, \dot{Z})$ be the unique solution to the BSDE

$$
\dot{Y}_{t}=\dot{Y}_{T}+\int_{t}^{T} \alpha\left(s, \dot{Y}_{s}, \mathbb{E} \dot{Y}_{s}, \dot{Z}_{s}\right) d s-\int_{t}^{T} \dot{Z}_{s} d B_{S}
$$

where

$$
\begin{aligned}
\alpha\left(t, \omega, y_{1}, y_{2}, z\right) & \\
& :=\left(\psi_{1} \wedge \psi_{2} \wedge \psi_{1}^{-} \wedge \psi_{2}^{-}\right)\left(t, \omega, y_{1}, y_{2}, z\right), \\
\dot{Y}_{T} & :=\left(\xi_{1}^{+}+b_{1}(T)\right) \wedge\left(\xi_{2}^{+}+b_{2}(T)\right) \wedge \xi_{1}^{-} \wedge \xi_{2}^{-} .
\end{aligned}
$$

By Proposition 4 it holds that

$$
\dot{Y}_{t} \leq L_{t}^{i}=Y_{t}^{+, i, 0}+b_{i}(t) .
$$

Hence,

$$
\dot{Y}_{t} \leq\left(Y_{t}^{+, i, 0}+b_{i}(t)\right) \wedge\left(\dot{Y}_{t}+\ell_{i}(t)\right),
$$

since $\ell_{i}(t)>0$ a.s.

Consider now the processes

$$
\begin{aligned}
& \left(Y^{-, i, 1}, Z^{-, i, 1}, K^{-, i, 1}\right)=F_{-}\left(\dot{Y}_{T}, \psi_{i}^{-}, S^{-, i, 1}\right), \\
& \left(Y^{+, i, 1}, Z^{+, i, 1}, K^{+, i, 1}\right)=F_{+}\left(\xi_{i}^{+}, \psi_{i}^{+}, S^{+, i, 1}\right),
\end{aligned}
$$

where

$$
\begin{gathered}
S_{t}^{-, i, 1}=\left(Y_{t}^{+, i, 0}+b_{i}(t)\right) \wedge\left(\dot{Y}_{t}+\ell_{i}(t)\right), \\
S_{t}^{+, 1,1}=\left(Y_{t}^{+, 2,0}-\ell_{1}(t)\right) \vee\left(Y_{t}^{-, 1,1}-a_{1}(t)\right), \\
S_{t}^{+, 2,1}=\left(Y_{t}^{+, 1,0}-\ell_{2}(t)\right) \vee\left(Y_{t}^{-, 2,1}-a_{2}(t)\right),
\end{gathered}
$$

and, for $n \geq 1$,

$$
\begin{aligned}
& \left(Y^{-, i, n+1}, Z^{-, i, n+1}, K^{-, i, n+1}\right)=F_{-}\left(\xi_{i}^{-}, \psi_{i}^{-}, S^{-, i, n+1}\right), \\
& \left(Y^{+, i, n+1}, Z^{+, i, n+1}, K^{+, i, n+1}\right)=F_{+}\left(\xi_{i}^{+}, \psi_{i}^{+}, S^{+, i, n+1}\right),
\end{aligned}
$$

where

$$
\begin{aligned}
& S_{t}^{-, 1, n+1}=\left(Y_{t}^{-, 2, n}+\ell_{1}(t)\right) \wedge\left(Y_{t}^{+, 1, n}+b_{1}(t)\right), \\
& S_{t}^{-, 2, n+1}=\left(Y_{t}^{-, 1, n}+\ell_{2}(t)\right) \wedge\left(Y_{t}^{+, 2, n}+b_{2}(t)\right), \\
& S_{t}^{+, 1, n+1}=\left(Y_{t}^{+, 2, n}-\ell_{1}(t)\right) \vee\left(Y_{t}^{-, 1, n+1}-a_{1}(t)\right), \\
& S_{t}^{+, 2, n+1}=\left(Y_{t}^{+, 1, n}-\ell_{2}(t)\right) \vee\left(Y_{t}^{-, 2, n+1}-a_{2}(t)\right) .
\end{aligned}
$$

Since $\left(Y^{+, i, 0}, Z^{+, i, 0}\right)$ is the solution of a standard MF-BSDE, the existence and uniqueness have been established in [6], and the existence and uniqueness of the processes $\left(Y^{-, i, 1}, Z^{-, i, 1}, K^{-, i, 1}\right)$ were established in Proposition 2. With this in mind, it is easily shown by the use of induction that for any $n \geq 1$ the triples $\left(Y^{+, i, n}, Z^{+, i, n}, K^{+, i, n}\right)$ exist, are unique, and belong to the appropriate spaces.

From Proposition 4 and the fact that $K_{T}^{+, i, 1}-K_{t}^{+, i, 1} \geq 0$ it follows that $Y_{t}^{+, i, 0} \leq Y_{t}^{+, i, 1}$. Moreover, by Proposition 4 again, it follows that $\dot{Y}_{t} \leq Y_{t}^{-, i, 1}$. Hence

$$
\begin{aligned}
\left(\dot{Y}_{t}+\ell_{1}(t)\right) & \wedge\left(Y_{t}^{+, 1,0}+b_{1}(t)\right) \\
& \leq\left(Y_{t}^{-, 2,1}+\ell_{1}(t)\right) \wedge\left(Y_{t}^{+, 1,1}+b_{1}(t)\right), \\
\left(\dot{Y}_{t}+\ell_{2}(t)\right) & \wedge\left(Y_{t}^{+, 2,0}+b_{2}(t)\right) \\
& \leq\left(Y_{t}^{-, 1,1}+\ell_{2}(t)\right) \wedge\left(Y_{t}^{+, 2,1}+b_{2}(t)\right) .
\end{aligned}
$$

Proposition 4 then yields $Y_{t}^{-, i, 1} \leq Y_{t}^{-, i, 2}$.

Now assume that

$$
\begin{aligned}
& Y_{t}^{+, i, n} \leq Y_{t}^{+, i, n+1}, \\
& Y_{t}^{-, i, n+1} \leq Y_{t}^{-, i, n+2} .
\end{aligned}
$$

Then

$$
\begin{aligned}
S_{t}^{+, 1, n+1} & =\left(Y_{t}^{+, 2, n}-\ell_{1}(t)\right) \vee\left(Y_{t}^{-, 1, n+1}-a_{1}(t)\right) \\
& \leq\left(Y_{t}^{+, 2, n+1}-\ell_{1}(t)\right) \vee\left(Y_{t}^{-, 1, n+2}-a_{1}(t)\right)=S_{t}^{+, 1, n+2}, \\
S_{t}^{+, 2, n+1} & =\left(Y_{t}^{+, 1, n}-\ell_{2}(t)\right) \vee\left(Y_{t}^{-, 2, n+1}-a_{2}(t)\right) \\
& \leq\left(Y_{t}^{+, 1, n+1}-\ell_{2}(t)\right) \vee\left(Y_{t}^{-, 2, n+2}-a_{2}(t)\right)=S_{t}^{+, 2, n+2},
\end{aligned}
$$

from which it follows, by Proposition 4 again, that $Y_{t}^{+, i, n+1} \leq$ $Y_{t}^{+, i, n+2}$. Hence, it also holds that

$$
\begin{aligned}
S_{t}^{-, 1, n+2} & =\left(Y_{t}^{-, 2, n+1}+\ell_{1}(t)\right) \wedge\left(Y_{t}^{+, 1, n+1}+b_{1}(t)\right) \\
& \leq\left(Y_{t}^{-, 2, n+2}+\ell_{1}(t)\right) \wedge\left(Y_{t}^{+, 1, n+2}+b_{1}(t)\right)=S_{t}^{-, 1, n+3}, \\
S_{t}^{-, 2, n+2} & =\left(Y_{t}^{-, 1, n+1}+\ell_{2}(t)\right) \wedge\left(Y_{t}^{+, 2, n+1}+b_{2}(t)\right) \\
& \leq\left(Y_{t}^{-, 1, n+2}+\ell_{2}(t)\right) \wedge\left(Y_{t}^{+, 2, n+2}+b_{2}(t)\right)=S_{t}^{-, 2, n+3} .
\end{aligned}
$$

Thus Proposition 4 yields that $Y_{t}^{-, i, n+2} \leq Y_{t}^{-, i, n+3}$. By induction,

$$
\begin{gathered}
Y_{t}^{+, i, n} \leq Y_{t}^{+, i, n+1}, \\
Y_{t}^{-, i, n+1} \leq Y_{t}^{-, i, n+2},
\end{gathered}
$$

for $n \geq 1$. 
By Proposition 3 these sequences are bounded, and since they are also increasing the limits exist. Denote these limits by

$$
\begin{aligned}
Y_{t}^{+, i} & :=\lim _{n \rightarrow \infty} Y_{t}^{+, i, n}, \\
Y_{t}^{-, i} & :=\lim _{n \rightarrow \infty} Y_{t}^{-, i, n} .
\end{aligned}
$$

In what follows we will prove that these limits are in fact continuous and solve the system (29). To do this we will use the following claim: there exists a positive constant $C$ such that, for all $n \geq 1$,

$$
\mathbb{E}\left[\int_{0}^{T}\left(\frac{d K_{t}^{-, i, n}}{d t}\right)^{2} d t\right] \leq C
$$

The first step towards proving this claim is to prove the absolute continuity of $d K_{t}^{-, i, 1}$ with respect to $d t$. Noting that

$$
S_{t}^{-, i, 1}=L_{t}^{i} \wedge\left(\dot{Y}_{t}+\ell_{i}(t)\right)=L_{t}^{i}-\left(L_{t}^{i}-\dot{Y}_{t}-\ell_{i}(t)\right)^{+},
$$

then in view of (A4) and the Itô-Tanaka formula, we get

$$
S_{t}^{-, i, 1}=S_{0}^{-, i, 1}+\int_{0}^{t} f_{i}(s) d s+\int_{0}^{t} g_{i}(s) d B_{s}-\frac{1}{2} \mathscr{L}_{t}^{i}
$$

where $\mathscr{L}^{i}$ is the local time at zero of the continuous semimartingale $L^{i}-\dot{Y}-\ell_{i}$ and where

$$
\begin{aligned}
f_{i}(t):= & -\psi_{i}\left(t, L_{t}^{i}, \mathbb{E} L_{t}^{i}, Z_{t}^{i}\right)+\mathbf{1}_{\left\{L_{t}^{i}>\dot{Y}_{t}+\ell_{i}(t)\right\}} \\
& \times\left(\psi_{i}\left(t, L_{t}^{i}, \mathbb{E} L_{t}^{i}, Z_{t}^{i}\right)-\alpha\left(t, \dot{Y}_{t}, \mathbb{E} \dot{Y}_{t}, \dot{Z}_{t}\right)+\bar{U}_{i}(t)\right), \\
g_{i}(t):= & Z_{t}^{i}-\mathbf{1}_{\left\{L_{t}^{i}>\dot{Y}_{t}+\ell_{i}(t)\right\}}\left(Z_{t}^{i}-\dot{Z}_{t}-\bar{V}_{i}(t)\right) .
\end{aligned}
$$

It follows that

$$
\begin{aligned}
& d\left(S_{t}^{-, i, 1}-Y_{t}^{-, i, 1}\right) \\
& =\left(f_{i}(t)+\psi_{i}^{-}\left(t, Y_{t}^{-, i, 1}, \mathbb{E} Y_{t}^{-, i, 1}, Z_{t}^{-, i, 1}\right)\right) d t \\
& \quad+\left(g_{i}(t)-Z_{t}^{-, i, 1}\right) d B_{t}-\frac{1}{2} d \mathscr{L}_{t}^{i}-d K_{t}^{-, i, 1}
\end{aligned}
$$

From Itô-Tanaka again we get that

$$
\begin{aligned}
d\left(S_{t}^{-, i, 1}-Y_{t}^{-, i, 1}\right)^{+} \\
=\mathbf{1}_{\left\{S_{t}^{-, i, 1}>Y_{t}^{-, i, 1}\right\}}\left(f_{i}(t)+\psi_{i}^{-}\left(t, Y_{t}^{-, i, 1}, \mathbb{E} Y_{t}^{-, i, 1}, Z_{t}^{-, i, 1}\right)\right) d t \\
\quad+\mathbf{1}_{\left\{S_{t}^{-, i, 1}>Y_{t}^{-, i, 1}\right\}}\left(g_{i}(t)-Z_{t}^{-, i, 1}\right) d B_{t} \\
\quad-\mathbf{1}_{\left\{S_{t}^{-, i, 1}>Y_{t}^{-, i, 1}\right\}}\left(d K_{t}^{-, i, 1}+\frac{1}{2} d \mathscr{L}_{t}^{i}\right)+\frac{1}{2} d \Lambda_{t}^{i},
\end{aligned}
$$

where $\Lambda_{t}^{i}$ is the local time at 0 for $S^{-, i, 1}-Y^{-, i, 1}$. Since $\left(S_{t}^{-, i, 1}-\right.$ $\left.Y_{t}^{-, i, 1}\right)^{+} \equiv S_{t}^{-, i, 1}-Y_{t}^{-, i, 1}$ the differentials must coincide, which yields that

$$
\begin{aligned}
\frac{1}{2} d \Lambda_{t}^{i}=\mathbf{1}_{\left\{Y_{t}^{-, i, 1}=S_{t}^{-, i, 1}\right\}}( & \left(f_{i}(t)+\psi_{i}^{-}\left(t, Y_{t}^{-, i, 1}, \mathbb{E} Y_{t}^{-, i, 1}, Z_{t}^{-, i, 1}\right)\right) d t \\
& \left.-\frac{1}{2} d \mathscr{L}_{t}^{i}-d K_{t}^{-, i, 1}\right) \\
=\mathbf{1}_{\left\{Y_{t}^{-, i, 1}=S_{t}^{-, i, 1}\right\}}( & \left(f_{i}(t)+\psi_{i}^{-}\left(t, Y_{t}^{-, i, 1}, \mathbb{E} Y_{t}^{-, i, 1}, Z_{t}^{-, i, 1}\right)\right) d t \\
& \left.-\frac{1}{2} d \mathscr{L}_{t}^{i}\right)-d K_{t}^{-, i, 1} .
\end{aligned}
$$

Hence

$$
\begin{aligned}
d K_{t}^{-, i, 1} & +\frac{1}{2}\left(d \Lambda_{t}^{i}+\mathbf{1}_{\left\{Y_{t}^{-, i, 1}=S_{t}^{-, i, 1}\right\}} d \mathscr{L}_{t}^{i}\right) \\
& =\mathbf{1}_{\left\{Y_{t}^{-, i, 1}=S_{t}^{-, i, 1}\right\}}\left(f_{i}(t)+\psi_{i}^{-}\left(t, Y_{t}^{-, i, 1}, \mathbb{E} Y_{t}^{-, i, 1}, Z_{t}^{-, i, 1}\right)\right) d t
\end{aligned}
$$

from which it follows that

$$
\begin{aligned}
0 & \leq d K_{t}^{-, i, 1} \\
& \leq \mathbf{1}_{\left\{Y_{t}^{-, i, 1}=S_{t}^{-, i, 1}\right\}}\left(f_{i}(t)+\psi_{i}^{-}\left(t, Y_{t}^{-, i, 1}, \mathbb{E} Y_{t}^{-, i, 1}, Z_{t}^{-, i, 1}\right)\right) d t \\
& \leq\left(\left|f_{i}(t)\right|+\left|\psi_{i}^{-}\left(t, Y_{t}^{-, i, 1}, \mathbb{E} Y_{t}^{-, i, 1}, Z_{t}^{-, i, 1}\right)\right|\right) d t .
\end{aligned}
$$

Now, in view of (A1)-(A4) and Proposition 3 there exists a constant $C$ such that

$$
\begin{gathered}
\mathbb{E}\left[\int _ { 0 } ^ { T } \left(\left|f_{i}(t)\right|^{2}+\left|\psi_{i}^{-}\left(t, Y_{t}^{-, i, 1}, \mathbb{E} Y_{t}^{-, i, 1}, Z_{t}^{-, i, 1}\right)\right|^{2}\right.\right. \\
\left.\left.+\left|g_{i}(t)\right|^{2}\right) d t\right] \leq C,
\end{gathered}
$$

which together with (62) proves (54) for $n=1$.

For the induction step, we only consider the case $i=1$ since the other case follows in a similar fashion. Assume that $d K_{t}^{-, i, n} / d t$ satisfies (54) and consider the obstacle process

$$
\begin{aligned}
S_{t}^{-, 1, n+1} & =\left(Y_{t}^{-, 2, n}+\ell_{1}(t)\right) \wedge\left(Y_{t}^{+, 1, n}+b_{1}(t)\right) \\
& =Y_{t}^{+, 1, n}+b_{1}(t)-\left(Y_{t}^{+, 1, n}+b_{1}(t)-Y_{t}^{-, 2, n}-\ell_{1}(t)\right)^{+} .
\end{aligned}
$$

By (A4) and the Itô-Tanaka formula, we get

$$
\begin{aligned}
S_{t}^{-, 1, n+1}= & S_{0}^{-, 1, n+1}+\int_{0}^{t} f_{n+1}(s) d s+\int_{0}^{t} g_{n+1}(s) d B_{s} \\
& -\frac{1}{2} \mathscr{L}_{t}^{1, n}-\mathbf{1}_{\left\{Y_{t}^{+, 1, n}+b_{1}(t) \leq Y_{t}^{-, 2, n}+\ell_{1}(t)\right\}} d K_{t}^{+, 1, n},
\end{aligned}
$$


where $\mathscr{L}^{1, n}$ is the local time at 0 for the continuous semimartingale $Y_{t}^{+, 1, n}+b_{1}(t)-Y_{t}^{-, 2, n}-\ell_{1}(t)$ and where

$$
\begin{aligned}
f_{n+1}(t):= & -\psi_{1}^{+}\left(t, Y_{t}^{+, 1, n}, \mathbb{E} Y_{t}^{+, 1, n}, Z_{t}^{+, 1, n}\right)+U_{1}(t) \\
& -\mathbf{1}_{\left\{Y_{t}^{+, 1, n}+b_{1}(t)>Y_{t}^{-, 2, n}+\ell_{1}(t)\right\}} \\
& \times\left(-\psi_{1}^{+}\left(t, Y_{t}^{+, 1, n}, \mathbb{E} Y_{t}^{+, 1, n}, Z_{t}^{+, 1, n}\right)+U_{1}(t)\right. \\
& \quad+\psi_{2}^{-}\left(t, Y_{t}^{-, 2, n}, \mathbb{E} Y_{t}^{-, 2, n}, Z_{t}^{-, 2, n}\right) \\
& \left.\quad-\bar{U}_{1}(t)-\frac{d K_{t}^{-, 2, n}}{d t}\right), \\
f_{n+1}(t):= & Z_{t}^{+, 1, n}+V_{1}(t) \\
& -\mathbf{1}_{\left\{Y_{t}^{+, 1, n}+b_{1}(t)>Y_{t}^{-, 2, n}+\ell_{1}(t)\right\}} \\
& \times\left(Z_{t}^{+, 1, n}+V_{1}(t)-Z_{t}^{-, 2, n}-\bar{V}_{1}(t)\right) .
\end{aligned}
$$

In view of (A1)-(A4) and Proposition 3, together with the induction assumption, there exists a constant $C>0$, independent of $n$, such that

$$
\begin{gathered}
\mathbb{E}\left[\int _ { 0 } ^ { T } \left(\left|f_{n+1}(t)\right|^{2}+\left|\psi_{1}^{-}\left(t, Y_{t}^{-, 1, n+1}, \mathbb{E} Y_{t}^{-, 1, n+1}, Z_{t}^{-, 1, n+1}\right)\right|^{2}\right.\right. \\
\left.\left.+\left|g_{n+1}(t)\right|^{2} d t\right)\right] \leq C .
\end{gathered}
$$

Following the same steps as we did earlier for $d K_{t}^{-, i, 1}$ it can be shown that

$$
\begin{aligned}
0 & \leq d K_{t}^{-, 1, n+1} \\
& \leq\left(\left|f_{n+1}(t)\right|+\left|\psi_{1}^{-}\left(t, Y_{t}^{-, 1, n+1}, \mathbb{E} Y_{t}^{-, 1, n+1}, Z_{t}^{-, 1, n+1}\right)\right|\right) d t,
\end{aligned}
$$

which yields that there exists a constant $C>0$ independent of $n$ such that

$$
\mathbb{E}\left[\int_{0}^{T}\left(\frac{d K_{t}^{-, 1, n+1}}{d t}\right)^{2} d t\right] \leq C .
$$

Hence claim (54) is true for all $n \geq 1$.

Proposition 3 and estimate (54) tell us that there is a subsequence along which the sequences of processes $\left(d K_{t}^{-, i, n} / d t\right),\left(\psi_{i}^{-}\left(t, Y_{t}^{-, i, n}, \mathbb{E} Y_{t}^{-, i, n}, Z_{t}^{-, i, n}\right)\right)$, and $\left(Z_{t}^{-, i, n}\right)$ converge weakly in their respective spaces $\mathscr{M}^{1,2}, \mathscr{M}^{1,2}$, and $\mathscr{M}^{d, 2}$ to the processes $\left(k_{t}^{-, i}, 0 \leq t \leq T\right),\left(\varphi_{t}^{-, i}, 0 \leq t \leq T\right)$, and $\left(Z_{t}^{-, i}, 0 \leq t \leq T\right)$.

Now for any $n \geq 0$ and any $\mathbb{F}$-stopping time $\tau$, we have

$$
\begin{aligned}
Y_{\tau}^{-, i, n}= & Y_{0}^{-, i, n}-\int_{0}^{\tau} \psi_{i}^{-}\left(s, Y_{s}^{-, i, n}, \mathbb{E} Y_{s}^{-, i, n}, Z_{s}^{-, i, n}\right) d s \\
& +K_{\tau}^{-, i, n}+\int_{0}^{\tau} Z_{s}^{-, i, n} d B_{s} .
\end{aligned}
$$

Taking the weak limits in each side of this equation along the subsequence mentioned earlier yields

$$
Y_{\tau}^{-, i}=Y_{0}^{-, i}-\int_{0}^{\tau} \varphi_{s}^{-, i} d s+\int_{0}^{\tau} k_{s}^{-, i} d s+\int_{0}^{\tau} Z_{s}^{-, i} d B_{s}, \quad \mathbb{P} \text {-a.s. }
$$

The processes on each side of the equality being optional, we can use the optional section theorem (see, e.g., [12, Theorem 86 , page 138]) to conclude that

$$
\begin{array}{r}
Y_{t}^{-, i}=Y_{0}^{-, i}-\int_{0}^{t} \varphi_{s}^{-, i} d s+\int_{0}^{t} k_{s}^{-, i} d s+\int_{0}^{t} Z_{s}^{-, i} d B_{s}, \\
\forall t \leq T, \quad \mathbb{P} \text {-a.s. }
\end{array}
$$

Therefore, the process $Y^{-, i}$ is continuous.

Relying on both Dini's theorem and Lebesgue's dominated convergence theorem we find that

$$
\lim _{n \rightarrow \infty} \mathbb{E}\left[\sup _{0 \leq t \leq T}\left|Y_{t}^{-, i, n}-Y_{t}^{-, i}\right|^{2}\right]=0 .
$$

In what follows we will characterize the limit processes $Y^{+, i}$ of the sequence $\left\{Y^{+, i, n}\right\}_{n \geq 0}$ as Snell envelopes of the processes in the sense that, $\mathbb{P}$-a.s. and for each $t \leq T$,

$$
\begin{array}{r}
Y_{t}^{+, i}=\underset{T \geq t}{\operatorname{ess} \sup } \mathbb{E}[ \\
{\left[\int_{t}^{\tau} \psi_{1}^{+}\left(s, Y_{s}^{+, i}, \mathbb{E} Y_{s}^{+, i}, Z_{s}^{+, i}\right) d s\right.} \\
\left.+\xi_{i}^{+} \mathbf{1}_{\{\tau=T\}}+S_{\tau}^{+, i} \mathbf{1}_{\{\tau<T\}} \mid \mathscr{F}_{t}\right],
\end{array}
$$

and then we derive their time continuity.

In view of Proposition 3 and applying Peng's monotone limit theorem (see [13]) to the sequence $\left(Y^{+, i, n}, Z^{+, i, n}, K^{+, i, n}\right)$, we obtain that $Y^{+, i}$ is càdlàg. Moreover, there exist a càdlàg nondecreasing process $K^{+, i} \in \mathscr{K}^{2}$ and a process $Z^{+, i} \in \mathscr{M}^{d, 2}$ such that

$$
\begin{aligned}
& Y_{t}^{+, i}=\xi_{i}+\int_{t}^{T} \psi_{i}^{+}\left(s, Y_{s}^{+, i}, \mathbb{E} Y_{s}^{+, i}, Z_{s}^{+, i}\right) d s+K_{T}^{+, i}-K_{t}^{+, i} \\
&-\int_{t}^{T} Z_{s}^{+, i} d B_{s}, \\
& Y_{t}^{+, i} \geq S_{t}^{+, i} .
\end{aligned}
$$

Hence, in view of Proposition 1 we arrive at (74) once we show that

$$
\int_{0}^{T}\left(Y_{t^{-}}^{+, i}-S_{t^{-}}^{+, i}\right) d K_{t}^{+, i}=0
$$


Consider the smallest $\psi_{i}^{+}$-supermartingale $\hat{Y}^{+, i}$ with lower obstacle $S^{+, i}$, which solves

$$
\begin{gathered}
\widehat{Y}_{t}^{+, i}=\xi_{i}^{+}+\int_{t}^{T} \psi_{i}^{+}\left(s, Y_{s}^{+, i}, \mathbb{E} Y_{s}^{+, i}, Z_{s}^{+, i}\right) d s \\
+\widehat{K}_{T}^{+, i}-\widehat{K}_{t}^{+, i}-\int_{t}^{T} \widehat{Z}_{s}^{+, i} d B_{s}, \\
\widehat{Y}_{t}^{+, i} \geq S_{t}^{+, i}, \\
\int_{0}^{T}\left(\widehat{Y}_{t^{-}}^{+, i}-S_{t^{-}}^{+, i}\right) d \widehat{K}_{t}^{+, i}=0 .
\end{gathered}
$$

By definition, we have that $Y_{t}^{+, i} \geq \widehat{Y}_{t}^{+, i}$. Using Proposition 5, we see that $\widehat{Y}_{t}^{+, i} \geq Y_{t}^{+, i, n}, n \geq 1$. Passing to the limit we get that $Y^{+, i}=\widehat{Y}^{+, i}, i=1,2$. Hence, (76) is satisfied.

It remains to show that $Y^{+, i}$ are continuous. Noting that $t>t^{-}$and that $Y^{+, i}$ satisfies (74), we find that $Y_{t}^{+, i} \leq Y_{t^{-}}^{+, i}$ or equivalently that $\Delta Y_{t}^{+, i} \leq 0$. Thus, it is sufficient to prove that the set $\left\{\Delta Y_{t}^{+, i}<0\right\}$ is empty.

By the Doob-Meyer decomposition of the Snell envelope (see Proposition 1) there exists, for each $i$, a continuous martingale $\left(M_{t}^{i}\right)_{0 \leq t \leq T}$, a continuous nondecreasing process $\left(C_{t}^{i}\right)_{0 \leq t \leq T}$, and a purely discontinuous process $\left(D_{t}^{i}\right)_{0 \leq t \leq T}$, with $C_{0}^{i}=D_{0}^{i}=0$, such that

$$
Y_{t}^{+, i}+\int_{0}^{t} \psi_{i}^{+}\left(s, Y_{s}^{+, i}, \mathbb{E} Y_{s}^{+, i}, Z_{s}^{+, i}\right) d s=M_{t}^{i}-C_{t}^{i}-D_{t}^{i}
$$

$\forall t \leq T$,

which means that $\Delta Y_{t}^{+, i}=-\Delta D_{t}^{i}$. Now in view of Proposition 1 we have the following properties of the jumps of $D^{i}$. If there is a jump at time $t$ in $D^{1}$, then this means that the process $\left(Y^{+, 2}-\ell_{2}\right) \vee\left(Y^{-, 1}-a_{1}\right)$ also jumps. Since $\ell_{1}$ and $Y^{-, 1}-a_{1}$ are continuous, this can only mean that there is a jump in $Y^{+, 2}$. This, in turn, means that there is a jump in $D^{2}$. And conversely, by the same type of reasoning, we can deduce that if there is a jump in $D^{2}$, there is also a jump in $D^{1}$. Hence, $D^{1}$ and $D^{2}$ always jump at the same time.

With this in mind, denote $X:=\left(Y^{+, 2}-\ell_{1}\right)$ and $F:=Y^{-, 1}$ $a_{1}$. We have

$$
\begin{aligned}
\{\Delta & (X \vee F)<0\} \\
& =\left\{X_{t} \vee F_{t}<X_{t^{-}} \vee F_{t}\right\} \\
& =\left\{\left\{X_{t} \vee F_{t}<X_{t^{-}} \vee F_{t}\right\} \cap\left\{X_{t}<F_{t}<X_{t^{-}}\right\}\right\} \\
& \cup\left\{\left\{X_{t} \vee F_{t}<X_{t^{-}} \vee F_{t}\right\} \cap\left\{X_{t}<X_{t^{-}}<F_{t}\right\}\right\} \\
& \cup\left\{\left\{X_{t} \vee F_{t}<X_{t^{-}} \vee F_{t}\right\} \cap\left\{F_{t}<X_{t}<X_{t^{-}}\right\}\right\} \\
& =\left\{\left\{F_{t}<X_{t^{-}}\right\} \cap\left\{X_{t}<F_{t}<X_{t^{-}}\right\}\right\} \\
& \cup\left\{\left\{F_{t}<F_{t}\right\} \cap\left\{X_{t}<X_{t^{-}}<F_{t}\right\}\right\} \\
& \cup\left\{\left\{X_{t}<X_{t^{-}}\right\} \cap\left\{F_{t}<X_{t}<X_{t^{-}}\right\}\right\}
\end{aligned}
$$

$$
\begin{aligned}
& =\left\{\left\{F_{t}<X_{t^{-}}\right\} \cap\left\{X_{t}<F_{t}\right\} \cap\left\{F_{t}<X_{t^{-}}\right\}\right\} \cup \emptyset \\
& \cup\left\{\left\{X_{t}<X_{t^{-}}\right\} \cap\left\{F_{t}<X_{t}\right\} \cap\left\{X_{t}<X_{t^{-}}\right\}\right\} \\
& =\left\{\left\{F_{t}<X_{t^{-}}\right\} \cap\left\{X_{t}<F_{t}\right\}\right\} \cup\left\{\left\{X_{t}<X_{t^{-}}\right\} \cap\left\{F_{t}<X_{t}\right\}\right\} \\
& =\left\{\left\{F_{t}<X_{t^{-}}\right\} \cap\left\{X_{t}<F_{t}\right\} \cap\left\{X_{t}<X_{t^{-}}\right\}\right\} \\
& \cup\left\{\left\{X_{t}<X_{t^{-}}\right\} \cap\left\{F_{t}<X_{t}\right\} \cap\left\{F_{t}<X_{t^{-}}\right\}\right\} \\
& =\left\{\left\{X_{t}<X_{t^{-}}\right\} \cap\left\{F_{t}<X_{t^{-}}\right\}\right\} \\
& \cap\left\{\left\{X_{t}<F_{t}\right\} \cup\left\{F_{t}<X_{t}\right\}\right\} \\
& =\left\{X_{t}<X_{t^{-}}\right\} \cap\left\{F_{t}<X_{t^{-}}\right\} \\
& =\left\{\Delta X_{t}<0\right\} \cap\left\{F_{t}<X_{t^{-}}\right\} \\
& =\left\{\Delta Y_{t}^{+, 2}<0,\left(Y_{t}^{-, 1}-a_{1}(t)\right)<\left(Y_{t^{-}}^{+, 2}-\ell_{1}(t)\right)\right\} .
\end{aligned}
$$

What this tells us is that if $S^{+, 1}$ jumps at time $t$, then we know two things. One is that $Y^{+, 2}$ also has a jump at time $t$ and the other is that

$$
Y_{t}^{-, 1}-a_{1}(t)<Y_{t^{-}}^{+, 2}-\ell_{1}(t) .
$$

Therefore, since $\Delta Y_{t}^{+, i}=-\Delta D_{t}^{i}$, in view of Proposition 1, we obtain that

$$
\begin{aligned}
\{\Delta & \left.D_{t}^{1}>0\right\} \\
& \subset\left\{\Delta D_{t}^{2}>0\right\} \cap\left\{Y_{t^{-}}^{+, 1}=S_{t^{-}}^{+, 1}\right\} \\
\quad & \left\{\Delta D_{t}^{2}>0\right\} \cap\left\{Y_{t^{-}}^{+, 1}=\left(Y_{t^{-}}^{+, 2}-\ell_{1}(t)\right) \vee\left(Y_{t}^{-, 1}-a_{1}(t)\right)\right\} \\
& =\left\{\Delta D_{t}^{2}>0\right\} \cap\left\{Y_{t^{-}}^{+, 1}=Y_{t^{-}}^{+, 2}-\ell_{1}(t)\right\} .
\end{aligned}
$$

Similarly, we have

$$
\begin{aligned}
\{\Delta & \left.D_{t}^{2}>0\right\} \\
& \subset\left\{\Delta D_{t}^{1}>0\right\} \cap\left\{Y_{t^{-}}^{+, 2}=S_{t^{-}}^{+, 2}\right\} \\
\quad & \left\{\Delta D_{t}^{1}>0\right\} \cap\left\{Y_{t^{-}}^{+, 2}=\left(Y_{t^{-}}^{+, 1}-\ell_{2}(t)\right) \vee\left(Y_{t}^{-, 2}-a_{2}(t)\right)\right\} \\
& =\left\{\Delta D_{t}^{1}>0\right\} \cap\left\{Y_{t^{-}}^{+, 2}=Y_{t^{-}}^{+, 1}-\ell_{2}(t)\right\} .
\end{aligned}
$$

Finally, since we know that $D^{1}$ and $D^{2}$ always jump at the same time, it holds that

$$
\begin{aligned}
\left\{\Delta D_{t}^{1}>0\right\} & \cup\left\{\Delta D_{t}^{1}>0\right\} \\
& =\left\{\Delta D_{t}^{1}>0\right\} \cap\left\{\Delta D_{t}^{2}>0\right\} \\
& =\left\{Y_{t^{-}}^{+, 1}=Y_{t^{-}}^{+, 2}-\ell_{1}(t)\right\} \\
& \cap\left\{Y_{t^{+}}^{+, 2}=Y_{t^{-}}^{+, 1}-\ell_{2}(t)\right\}=\emptyset,
\end{aligned}
$$


since, for any $t \leq T, \ell_{1}(t)+\ell_{2}(t)>0$. It follows that the processes $D^{1}$ and $D^{2}$ are constant and identically equal to 0 , since $D_{0}^{1}=D_{0}^{2}=0$. Hence, the processes $Y^{+, 1}$ and $Y^{+, 2}$ are continuous. This, in turn, given (75), yields the continuity of the increasing processes $K^{+, i}$. Therefore, $\left(Y^{+, i}, Z^{+, i}, K^{+, i}\right)$ is a solution to the first part of the system (29).

By Dini's theorem and Lebesgue's dominated convergence theorem again we also conclude that the convergence of $\left(Y^{+, 1, n}\right)_{n \geq 1}$ to $Y^{+, 1}$ holds in $S_{c}^{2}$; that is,

$$
\lim _{n \rightarrow \infty} \mathbb{E}\left[\sup _{0 \leq t \leq T}\left|Y_{t}^{+, i, n}-Y_{t}^{+, i}\right|^{2}\right]=0 \text {. }
$$

Furthermore, since $Y^{-, i}$ is continuous, we can rely on standard arguments, in particular, by applying Itô's formula to $\left(Y^{+, i, n}-Y^{+, i, m}\right)^{2},(m, n \geq 0)$, to claim that $\left(Z^{+, i, n}\right)_{\geq 0}$ is a Cauchy sequence, and therefore it converges to $Z^{+, i}$ in $\mathscr{M}^{d, 2}$; that is,

$$
\lim _{n \rightarrow \infty} \mathbb{E}\left[\int_{0}^{T}\left|Z_{t}^{+, i, n}-Z_{t}^{+, i}\right|^{2} d t\right]=0 .
$$

Combining this with (84) and the definition of the processes $Y^{+, i, n}$ and $Y^{+, i}$ yields that

$$
\lim _{n \rightarrow \infty} \mathbb{E}\left[\sup _{0 \leq t \leq T}\left|K_{t}^{+, i, n}-K_{t}^{+, i}\right|^{2}\right]=0 .
$$

In total, we have shown that

$$
\begin{aligned}
\lim _{n \rightarrow \infty} \mathbb{E} & {\left[\sup _{0 \leq t \leq T}\left|Y_{t}^{+, i, n}-Y_{t}^{+, i}\right|^{2}\right.} \\
& \left.+\int_{0}^{T}\left|Z_{t}^{+, i, n}-Z_{t}^{+, i}\right|^{2} d t+\sup _{0 \leq t \leq T}\left|K_{t}^{+, i, n}-K_{t}^{+, i}\right|^{2}\right]=0 .
\end{aligned}
$$

Using (84) and applying Itô's formula to $\left(Y^{-, i, n}-Y^{-, i, m}\right)^{2}$, $(m, n \geq 0)$, it follows that $\left(Z^{-, i, n}\right)_{n \geq 1}$ is a Cauchy sequence and therefore converges to $Z^{-, i}$ in $\mathscr{M}^{d, 2}$; that is,

$$
\lim _{n \rightarrow \infty} \mathbb{E}\left[\int_{0}^{T}\left|Z_{t}^{-, i, n}-Z_{t}^{-, i}\right|^{2} d t\right]=0 .
$$

Using this and taking into account the decomposition obtained in (72), we arrive at

$$
\begin{gathered}
Y_{t}^{-, i}=\xi_{i}^{-}+\int_{t}^{T} \psi_{i}^{-}\left(s, Y_{s}^{-, i}, \mathbb{E} Y_{s}^{-, i}, Z_{s}^{-, i}\right) d s \\
-\int_{t}^{T} k_{s}^{-, i} d s-\int_{t}^{T} Z_{s}^{-, i} d B_{s}, \\
Y_{t}^{-, 1} \leq\left(Y_{t}^{-, 2}+\ell_{1}(t)\right) \wedge\left(Y_{t}^{+, 1}+b_{1}(t)\right), \\
Y_{t}^{-, 2} \leq\left(Y_{t}^{-, 1}+\ell_{2}(t)\right) \wedge\left(Y_{t}^{+, 2}+b_{2}(t)\right) .
\end{gathered}
$$

As shown above, it also holds that

$$
\begin{gathered}
\lim _{n \rightarrow \infty} \mathbb{E}\left[\sup _{0 \leq t \leq T}\left|Y_{t}^{-, i, n}-Y_{t}^{-, i}\right|^{2}+\int_{0}^{T}\left|Z_{t}^{-, i, n}-Z_{t}^{-, i}\right|^{2} d t\right. \\
\left.+\sup _{0 \leq t \leq T}\left|K_{t}^{-, i, n}-\int_{0}^{t} k_{s}^{-, i} d s\right|^{2}\right]=0 .
\end{gathered}
$$

Moreover, due to the weak convergence of $\left(d K_{t}^{-, i, n} / d t, 0 \leq\right.$ $t \leq T, n \geq 1)$ to the process $k^{-, i}$ and the strong convergence of $\left(Y^{+, i, n}\right)$ and $\left(Y^{-, i, n}\right)$ in $\mathcal{S}^{2}$, it follows that

$$
0=\int_{0}^{T}\left(S_{t}^{-, i, n}-Y_{t}^{-, i, n}\right) d K_{t}^{-, i, n} \longrightarrow \int_{0}^{T}\left(S_{t}^{-, i}-Y_{t}^{-, i}\right) k_{t}^{-, i} d s
$$

as $n \rightarrow \infty$, which implies that if we define

$$
K_{t}^{-, i}:=\int_{0}^{t} k_{s}^{-, i} d s, \quad t \leq T,
$$

then $\left(Y^{-, i}, Z^{-, i}, K^{-, i}\right)$ is a solution to the second part of (29).

It remains to show that the obtained solution is minimal. To do this, we compare $Y^{+, i}$ with the smallest $\psi_{i}^{+}$supermartingale with lower obstacle $S^{+, i}$, denoted by $\underline{Y}^{+, i}$, and we compare $Y^{+, i}$ with the smallest $\psi_{i}^{-}$-supermartingale with upper obstacle $S^{-, i}$, denoted by $\underline{Y}^{-, i}$; that is, let $\left(\underline{Y}^{ \pm, i}, \underline{Z}^{ \pm, i}, \underline{K}^{ \pm, i}\right)$ be the smallest solution to the system

$$
\begin{array}{ll}
\left(\underline{Y}^{+, i}, \underline{Z}^{+, i}, \underline{K}^{+, i}\right)=F_{+}\left(\xi_{i}^{+}, \psi_{i}^{+}, S^{+, i}\right), \quad i=1,2, \\
\left(\underline{Y}^{-, i}, \underline{Z}^{-, i}, \underline{K}^{-, i}\right)=F_{-}\left(\xi_{i}^{-}, \psi_{i}^{-}, S^{-, i}\right), \quad i=1,2 .
\end{array}
$$

Using Proposition 4 and the minimality of the solution we can conclude that $\underline{Y}^{ \pm, i} \leq Y^{ \pm, i}$. On the other hand, Proposition 4 also tells us that for all $n \geq 0$ we have $Y^{ \pm, i, n} \leq$ $\underline{Y}^{ \pm, i}$. Now since $Y^{ \pm, i, n}$ are increasing in $n$, we can take the limit $n \rightarrow \infty$ and obtain that $Y^{ \pm, i} \leq \underline{Y}^{ \pm, i}$. Thus, $\underline{Y}^{ \pm, i}=Y^{ \pm, i}$.

Finally to establish that the solution is not unique in general, the counterexample found in [1] is valid here as well.

\section{Appendices}

\section{A. Proof of Proposition 2}

The proof uses techniques found in $[2,7,8]$. Let $\mathbf{S}$ be the space of progressively measurable $\left\{\left(Y_{t}, Z_{t}\right): 0 \leq t \leq T\right\}$ taking values in $\mathbb{R} \times \mathbb{R}^{d}$ such that $Z \in \mathscr{M}^{d, 2}$ and $Y_{t} \geq S_{t}$ for $0 \leq t \leq T$.

Take any $(U, V) \in \mathbf{S}$ and define $\tilde{f}(t):=f\left(t, U_{t}, \mathbb{E} U_{t}, V_{t}\right)$. Then, by Proposition 5.1 in [2], there is a unique triplet $(Y, Z, K)$ such that

$$
\begin{gathered}
Y \in \mathcal{S}^{2}, \quad Z \in \mathscr{M}^{d, 2}, \quad K \in \mathscr{K}^{2}, \quad K_{T} \in L^{2}\left(\Omega, \mathscr{F}_{T}, \mathbb{P}\right) \\
Y_{t}=\xi+\int_{t}^{T} \tilde{f}(s) d s+K_{T}-K_{t}-\int_{t}^{T} Z_{s} d B_{s}, \quad 0 \leq t \leq T, \\
Y_{t} \geq S_{t}, \quad 0 \leq t \leq T, \\
K_{0}=0, \quad \int_{0}^{T}\left(Y_{t}-S_{t}\right) d K_{t}=0 .
\end{gathered}
$$

We define a mapping $\Phi$ from $\mathbf{S}$ onto itself by saying that $(Y, Z)=\Phi(U, V)$ is the unique element of $\mathbf{S}$ such that if we define

$$
K_{t}=Y_{t}-Y_{0}-\int_{0}^{t} \tilde{f}(s) d s+\int_{0}^{t} Z_{s} d B_{s}, \quad 0 \leq t \leq T,
$$


then $(Y, Z, K)$ is the unique triplet which solves the system above.

Now let $\left(U^{\prime}, V^{\prime}\right)$ be another element of $S$ and let $\left(Y^{\prime}, Z^{\prime}\right)=$ $\Phi\left(U^{\prime}, V^{\prime}\right)$. In addition, let

$$
\begin{gathered}
\bar{U}=U-U^{\prime}, \quad \bar{V}=V-V^{\prime}, \quad \bar{Y}=Y-Y^{\prime}, \\
\bar{Z}=Z-Z^{\prime}, \quad \bar{f}=\tilde{f}-\tilde{f}^{\prime},
\end{gathered}
$$

where $\tilde{f}^{\prime}(t):=f\left(t, U_{t}^{\prime}, \mathbb{E} U_{t}^{\prime}, V_{t}^{\prime}\right)$.

For any $\beta>0$, applying Itô's formula to $e^{\beta t} \bar{Y}_{t}^{2}$ and then taking expectations we find that

$$
\begin{array}{r}
\mathbb{E}\left[e^{\beta t} \bar{Y}_{t}^{2}+\int_{t}^{T} \beta e^{\beta s} \bar{Y}_{s}^{2} d s+\int_{t}^{T} e^{\beta s}\left|\bar{Z}_{s}\right|^{2} d s\right] \\
=2 \mathbb{E}\left[\int_{t}^{T} e^{\beta s} \bar{Y}_{s} \bar{f}(s) d s+\int_{t}^{T} e^{\beta s} \bar{Y}_{s} d \bar{K}_{s}\right] .
\end{array}
$$

Moreover, since

$$
\begin{aligned}
2 \mathbb{E} & {\left[\int_{t}^{T} e^{\beta s} \bar{Y}_{s} d \bar{K}_{s}\right] } \\
= & 2 \mathbb{E}\left[\int_{t}^{T} e^{\beta s}\left(Y_{s}-Y_{s}^{\prime}\right) d\left(K_{s}-K_{s}^{\prime}\right)\right] \\
= & 2 \mathbb{E}\left[\int_{t}^{T} e^{\beta s}\left[\left(Y_{s}-S_{s}\right)-\left(Y_{s}^{\prime}-S_{s}^{\prime}\right)\right]\left(d K_{s}-d K_{s}^{\prime}\right)\right] \\
= & -2 \mathbb{E}\left[\int_{t}^{T} e^{\beta s}\left(Y_{s}-S_{s}\right) d K_{s}^{\prime}\right] \\
& -2 \mathbb{E}\left[\int_{t}^{T} e^{\beta s}\left(Y_{s}^{\prime}-S_{s}^{\prime}\right) d K_{s}\right] \leq 0,
\end{aligned}
$$

it holds that

$$
\begin{gathered}
\mathbb{E}\left[\int_{0}^{T} \beta e^{\beta s} \bar{Y}_{s}^{2} d s+\int_{0}^{T} e^{\beta s}\left|\bar{Z}_{s}\right|^{2} d s\right] \\
\leq 2 \mathbb{E}\left[\int_{0}^{T} e^{\beta s} \bar{Y}_{s} \bar{f}(s) d s\right] .
\end{gathered}
$$

Using the Lipschitz property of $f$ and then Young's inequality yields that

$$
\begin{aligned}
& \mathbb{E} {\left[\int_{0}^{T} \beta e^{\beta s} \bar{Y}_{s}^{2} d s+\int_{0}^{T} e^{\beta s}\left|\bar{Z}_{s}\right|^{2} d s\right] } \\
& \leq 2 \mathbb{E}\left[\int_{0}^{T} e^{\beta s} \bar{Y}_{s} \bar{f}(s) d s\right] \\
&=2 \mathbb{E}\left[\int _ { 0 } ^ { T } e ^ { \beta s } \overline { Y } _ { s } \left(f\left(s, U_{s}, \mathbb{E} U_{s}, V_{s}\right)\right.\right. \\
&\left.\left.-f\left(s, U_{s}^{\prime}, \mathbb{E} U_{s}^{\prime}, V_{s}^{\prime}\right)\right) d s\right]
\end{aligned}
$$

$$
\begin{aligned}
& \leq 2 K \mathbb{E}\left[\int _ { 0 } ^ { T } e ^ { \beta s } \overline { Y } _ { s } \left(\left|U_{s}-U_{s}^{\prime}\right|+\left|\mathbb{E} U_{s}-\mathbb{E} U_{s}^{\prime}\right|\right.\right. \\
& \left.\left.+\left|V_{s}-V_{s}^{\prime}\right|\right) d s\right] \\
& \leq 2 K \mathbb{E}\left[\int _ { 0 } ^ { T } e ^ { \beta s } \overline { Y } _ { s } \left(\left|U_{s}-U_{s}^{\prime}\right|+\mathbb{E}\left|U_{s}-U_{s}^{\prime}\right|\right.\right. \\
& \left.\left.+\left|V_{s}-V_{s}^{\prime}\right|\right) d s\right] \\
& \leq 12 K^{2} \mathbb{E}\left[\int_{0}^{T} e^{\beta s} \bar{Y}_{s}^{2} d s\right] \\
& +\frac{1}{12} \mathbb{E}\left[\int _ { 0 } ^ { T } e ^ { \beta s } \left(\left|U_{s}-U_{s}^{\prime}\right|+\mathbb{E}\left|U_{s}-U_{s}^{\prime}\right|\right.\right. \\
& +12 K^{2} \mathbb{E}\left[\int_{0}^{T} e^{\beta s} \bar{Y}_{s}^{2} d s\right] \\
& +\frac{1}{4} \mathbb{E}\left[\int _ { 0 } ^ { T } e ^ { \beta s } \left(\left|U_{s}-U_{s}^{\prime}\right|^{2}+\mathbb{E}\left|U_{s}-U_{s}^{\prime}\right|^{2}\right.\right. \\
& +\frac{1}{2} \mathbb{E}\left[\int_{0}^{T} e^{\beta s}\left(\bar{U}_{s}^{2}+\left|\bar{V}_{s}\right|^{2}\right) d s\right] . \\
& +\frac{1}{4} \int_{0}^{T} e^{\beta s}\left(2 \mathbb{E}_{U_{s}^{2}}^{2} \mathbb{E}\left|\bar{V}_{s}\right|^{2}\right) d s \\
& =12 K^{2} \mathbb{E}\left[\int_{0}^{T} e^{\beta s} \bar{Y}_{s}^{2} d s\right] \\
& \left.\left.+\left|V_{s}-V_{s}^{\prime}\right|^{2}\right) d s\right]
\end{aligned}
$$

Setting $\beta=12 K^{2}+1$ we find

$$
\begin{aligned}
& \mathbb{E}\left[\int_{0}^{T} e^{\beta s}\left(\bar{Y}_{s}^{2}+\left|\bar{Z}_{s}\right|^{2}\right) d s\right] \\
& \quad \leq \frac{1}{2} \mathbb{E}\left[\int_{0}^{T} e^{\beta s}\left(\bar{U}_{s}^{2}+\left|\bar{V}_{s}\right|^{2}\right) d s\right] .
\end{aligned}
$$

Thus, the mapping $\Phi$ is a strict contraction on $\mathbf{S}$ with the norm

$$
\|(Y, Z)\|_{\beta}=\left(\mathbb{E} \int_{0}^{T} e^{\beta s}\left(Y_{s}^{2}+\left|Z_{s}\right|^{2}\right) d s\right)^{1 / 2} .
$$

The Banach fixed point theorem then yields that $\Phi$ has a unique fixed point, which is the sought solution. 


\section{B. Proof of Proposition 3}

Applying Itô's formula to $Y_{t}^{2}$ yields that

$$
\begin{aligned}
Y_{t}^{2}+\int_{t}^{T}\left|Z_{s}\right|^{2} d s \\
=\xi^{2}+2 \int_{t}^{T} Y_{s} f\left(s, Y_{s}, \mathbb{E} Y_{s}, Z_{s}\right) d s \\
\quad+2 \int_{t}^{T} Y_{s} d K_{s}-2 \int_{t}^{T} Y_{s} Z_{s} d B_{s} \\
=\xi^{2}+2 \int_{t}^{T} Y_{s} f\left(s, Y_{s}, \mathbb{E} Y_{s}, Z_{s}\right) d s \\
\quad+2 \int_{t}^{T} S_{s} d K_{s}-2 \int_{t}^{T} Y_{s} Z_{s} d B_{s},
\end{aligned}
$$

and so, by taking expectations, we get

$$
\begin{aligned}
\mathbb{E} & {\left[Y_{t}^{2}+\int_{t}^{T}\left|Z_{s}\right|^{2} d s\right] } \\
& =\mathbb{E}\left[\xi^{2}+2 \int_{t}^{T} S_{s} d K_{s}\right]+\mathbb{E}\left[2 \int_{t}^{T} Y_{s} f\left(s, Y_{s}, \mathbb{E} Y_{s}, Z_{s}\right) d s\right] .
\end{aligned}
$$

Using the Lipschitz property of $f$ yields that

$$
\begin{aligned}
& \mathbb{E}\left[2 \int_{t}^{T} Y_{s} f\left(s, Y_{s}, \mathbb{E} Y_{s}, Z_{t}\right) d s\right] \\
& \leq \mathbb{E}\left[\int_{t}^{T} 2\left|Y_{s}\right||f(s, 0,0,0)|+2 K\left|Y_{s}\right|^{2}\right. \\
& \left.\quad+2 K\left|Y_{s}\right| \mathbb{E}\left[\left|Y_{s}\right|\right]+2 K\left|Y_{s}\right|\left|Z_{s}\right| d s\right] \\
& \leq \int_{t}^{T} \mathbb{E}\left[\left|Y_{s}\right|^{2}\right]+\mathbb{E}\left[|f(s, 0,0,0)|^{2}\right]+2 K \mathbb{E}\left[\left|Y_{s}\right|^{2}\right] \\
& \quad+2 K \mathbb{E}\left[\left|Y_{s}\right|\right]^{2}+2 K^{2} \mathbb{E}\left[\left|Y_{s}\right|^{2}\right]+\frac{1}{2} \mathbb{E}\left[\left|Z_{s}\right|^{2}\right] d s \\
& \leq \int_{t}^{T}\left(1+4 K+2 K^{2}\right) \mathbb{E}\left[\left|Y_{s}\right|^{2}\right]+\mathbb{E}\left[\left|f(s, 0,0,0)^{2}\right|\right] d s \\
& +\frac{1}{2} \int_{t}^{T} \mathbb{E}\left[\left|Z_{s}\right|^{2}\right] d s .
\end{aligned}
$$

Hence

$$
\begin{aligned}
\mathbb{E}\left[\left|Y_{t}\right|^{2}\right]+\frac{1}{2} \mathbb{E}\left[\int_{t}^{T}\left|Z_{s}\right|^{2} d s\right] \\
\leq \mathbb{E}\left[\xi^{2}+2 \int_{t}^{T} S_{s} d K_{s}+\int_{t}^{T}|f(s, 0,0,0)|^{2} d s\right] \\
+\left(1+4 K+2 K^{2}\right) \int_{t}^{T} \mathbb{E}\left[\left|Y_{s}\right|^{2}\right] d s
\end{aligned}
$$

Therefore,

$$
\begin{gathered}
\mathbb{E}\left[\left|Y_{t}\right|^{2}\right] \leq \mathbb{E}\left[\xi^{2}+2 \int_{t}^{T} S_{s} d K_{s}+\int_{t}^{T}|f(s, 0,0,0)|^{2} d s\right] \\
+\left(1+4 K+2 K^{2}\right) \int_{t}^{T} \mathbb{E}\left[\left|Y_{s}\right|^{2}\right] d s \\
\frac{1}{2} \mathbb{E}\left[\int_{t}^{T}\left|Z_{s}\right|^{2} d s\right] \\
\leq \mathbb{E}\left[\xi^{2}+2 \int_{t}^{T} S_{s} d K_{s}+\int_{t}^{T}|f(s, 0,0,0)|^{2} d s\right] \\
+\left(1+4 K+2 K^{2}\right) \int_{t}^{T} \mathbb{E}\left[\left|Y_{s}\right|^{2}\right] d s \\
\leq \mathbb{E}\left[\xi^{2}+2 \int_{t}^{T} S_{s}^{+} d K_{s}+\int_{t}^{T}|f(s, 0,0,0)|^{2} d s\right] \\
+\left(1+4 K+2 K^{2}\right) \int_{t}^{T} \mathbb{E}\left[\left|Y_{s}\right|^{2}\right] d s .
\end{gathered}
$$

Using Gronwall's inequality on (B.5), we obtain that

$$
\begin{aligned}
\mathbb{E}\left[\left|Y_{t}\right|^{2}\right] & \leq C \mathbb{E}\left[\xi^{2}+2 \int_{t}^{T} S_{s} d K_{s}+\int_{t}^{T}|f(s, 0,0,0)|^{2} d s\right] \\
& \leq C \mathbb{E}\left[\xi^{2}+2 \int_{t}^{T} S_{s}^{+} d K_{s}+\int_{t}^{T}|f(s, 0,0,0)|^{2} d s\right] .
\end{aligned}
$$

Inserting into (B.6) yields that

$$
\begin{aligned}
\frac{1}{2} \mathbb{E} & {\left[\int_{t}^{T}\left|Z_{s}\right|^{2} d s\right] } \\
\leq & \mathbb{E}\left[\xi^{2}+2 \int_{t}^{T} S_{s}^{+} d K_{s}+\int_{t}^{T}|f(s, 0,0,0)|^{2} d s\right] \\
& +\left(1+4 K+2 K^{2}\right) \\
& \times \int_{t}^{T} C \mathbb{E}\left[\xi^{2}+2 \int_{s}^{T} S_{u}^{+} d K_{u}+\int_{s}^{T}|f(u, 0,0,0)|^{2} d u\right] d s \\
\leq & \mathbb{E}\left[\xi^{2}+2 \int_{t}^{T} S_{s}^{+} d K_{s}+\int_{t}^{T}|f(s, 0,0,0)|^{2} d s\right] \\
& +\left(1+4 K+2 K^{2}\right) \\
& \times C \mathbb{E}\left[T\left(\xi^{2}+2 \int_{0}^{T} S_{s}^{+} d K_{s}+\int_{0}^{T}|f(s, 0,0,0)|^{2} d s\right)\right] .
\end{aligned}
$$

Hence

$$
\begin{aligned}
& \mathbb{E}\left[\int_{0}^{T}\left|Z_{s}\right|^{2} d s\right] \\
& \quad \leq C^{\prime} \mathbb{E}\left[\xi^{2}+2 \int_{0}^{T} S_{s}^{+} d K_{s}+\int_{0}^{T}|f(s, 0,0,0)|^{2} d s\right] .
\end{aligned}
$$


Consider now the equality

$$
K_{T}=Y_{0}-\xi-\int_{0}^{T} f\left(t, Y_{t}, \mathbb{E} Y_{t}, Z_{t}\right) d t+\int_{0}^{T} Z_{t} d B_{t} .
$$

We have

$$
\begin{aligned}
\mathbb{E}\left[K_{T}^{2}\right]=\mathbb{E}\left[\left(Y_{0}-\xi-\int_{0}^{T} f\left(t, Y_{t}, \mathbb{E} Y_{t}, Z_{t}\right) d t\right.\right. & \\
& \left.\left.\quad \int_{0}^{T} Z_{t} d B_{t}\right)^{2}\right] \\
\leq & 4 \mathbb{E}\left[Y_{0}^{2}+\xi^{2}+\left(\int_{0}^{T} f\left(t, Y_{t}, \mathbb{E} Y_{t}, Z_{t}\right) d t\right)^{2}\right. \\
& \left.+\left(\int_{0}^{T} Z_{t} d B_{t}\right)^{2}\right] \\
= & \{\text { Itồs rule on the squared stochastic integral }\} \\
= & 4 \mathbb{E}\left[Y_{0}^{2}+\xi^{2}+\left(\int_{0}^{T} f\left(t, Y_{t}, \mathbb{E} Y_{t}, Z_{t}\right) d t\right)^{2}\right. \\
& \left.+\int_{0}^{T}\left|Z_{t}\right|^{2} d t\right] \\
\leq & 4 \mathbb{E}\left[Y_{0}^{2}+\xi^{2}+\int_{0}^{T} f\left(t, Y_{t}, \mathbb{E} Y_{t}, Z_{t}\right)^{2} d t\right. \\
& \left.+\int_{0}^{T}\left|Z_{t}\right|^{2} d t\right] \\
& +4 \mathbb{E}\left[\xi^{2}+\int_{0}^{T} f\left(t, Y_{t}, \mathbb{E} Y_{t}, Z_{t}\right)^{2} d t\right], \\
\leq & {\left[\xi^{2}+2 \int_{0}^{T} S_{t}^{+} d K_{t}+\int_{0}^{T}|f(t, 0,0,0)|^{2} d t\right] }
\end{aligned}
$$

where the last inequality follows from using (B.7) and (B.9). Now from the Lipschitz property of $f$ we get

$$
\begin{aligned}
& \mathbb{E}\left[\int_{0}^{T} f\left(t, Y_{t}, \mathbb{E} Y_{t}, Z_{t}\right)^{2} d t\right] \\
& \quad \leq K^{2} \int_{0}^{T} \mathbb{E}\left[\left(|f(t, 0,0,0)|+\left|Y_{t}\right|+\left|\mathbb{E} Y_{t}\right|+\left|Z_{t}\right|\right)^{2}\right] d t \\
& \quad \leq 4 K^{2} \int_{0}^{T} \mathbb{E}\left[|f(t, 0,0,0)|^{2}+\left|Y_{t}\right|^{2}+\left|\mathbb{E} Y_{t}\right|^{2}+\left|Z_{t}\right|^{2}\right] d t \\
& \quad \leq 4 K^{2} \int_{0}^{T} \mathbb{E}\left[|f(t, 0,0,0)|^{2}+\left|Y_{t}\right|^{2}+\mathbb{E}\left[\left|Y_{t}\right|^{2}\right]+\left|Z_{t}\right|^{2}\right] d t
\end{aligned}
$$

$$
\begin{aligned}
= & 4 K^{2} \mathbb{E}\left[\int_{0}^{T}|f(t, 0,0,0)|^{2} d t\right] \\
& +8 K^{2} \mathbb{E}\left[\int_{0}^{T}\left|Y_{t}\right|^{2} d t\right]+4 K^{2} \mathbb{E}\left[\int_{0}^{T}\left|Z_{t}\right|^{2} d t\right],
\end{aligned}
$$

which, by inserting into the above and then using (B.7) and (B.9) again, yields that

$$
\begin{aligned}
\mathbb{E} & {\left[K_{T}^{2}\right] } \\
\leq & C \mathbb{E}\left[\xi^{2}+2 \int_{0}^{T} S_{s}^{+} d K_{s}+\int_{0}^{T}|f(s, 0,0,0)|^{2} d s\right] \\
& +4 \mathbb{E}\left[\xi^{2}\right]+8 K^{2} \mathbb{E}\left[\int_{0}^{T}|f(t, 0,0,0)|^{2} d t\right] \\
\leq & C \mathbb{E}\left[\xi^{2}+2 \int_{0}^{T} S_{s}^{+} d K_{s}+\int_{0}^{T}|f(s, 0,0,0)|^{2} d s\right] \\
& +8\left(1+K^{2}\right) \mathbb{E}\left[\xi^{2}+\int_{0}^{T}|f(t, 0,0,0)|^{2} d t+2 \int_{0}^{T} S_{t}^{+} d K_{t}\right] \\
= & C \mathbb{E}\left[\xi^{2}+\int_{0}^{T}|f(s, 0,0,0)|^{2} d s+2 \int_{0}^{T} S_{s}^{+} d K_{s}\right] \\
\leq & C \mathbb{E}\left[\xi^{2}+\int_{0}^{T}|f(s, 0,0,0)|^{2} d s\right] \\
& +\mathbb{E}\left[2 C\left(\sup _{0 \leq t \leq T} S_{s}^{+}\right) K_{T}\right] \\
\leq & C \mathbb{E}\left[\xi^{2}+\int_{0}^{T}|f(s, 0,0,0)|^{2} d s\right] \\
& +2 C^{2} \mathbb{E}\left[\sup _{0 \leq t \leq T}\left(S_{s}^{+}\right)^{2}\right]+\frac{1}{2} \mathbb{E}\left[K_{T}^{2}\right] .
\end{aligned}
$$

Thus

$$
\mathbb{E}\left[K_{T}^{2}\right] \leq C\left[\xi^{2}+\int_{0}^{T}|f(s, 0,0,0)|^{2} d s+\sup _{0 \leq t \leq T}\left(S_{s}^{+}\right)^{2}\right] .
$$

Using the Burkholder-Davis-Gundy inequality we find that

$$
\mathbb{E}\left[\sup _{0 \leq t \leq T}\left|Y_{t}\right|^{2}\right] \leq C \mathbb{E}\left[\int_{0}^{T}\left|Z_{t}\right|^{2} d t\right] .
$$

Hence, again in view of (B.9), we get

$$
\begin{aligned}
& \mathbb{E}\left[\sup _{0 \leq t \leq T}\left|Y_{t}\right|^{2}+\int_{0}^{T}\left|Z_{t}\right|^{2} d t+\left|K_{T}\right|^{2}\right] \\
& \leq \mathbb{E}\left[(1+C) \int_{0}^{T}\left|Z_{t}\right|^{2} d t+\left|K_{T}\right|^{2}\right] \\
& \leq C \mathbb{E}\left[\xi^{2}+\int_{0}^{T}|f(s, 0,0,0)|^{2} d s+\sup _{0 \leq t \leq T}\left(S_{s}^{+}\right)^{2}\right] .
\end{aligned}
$$




\section{Proof of Proposition 4}

Itô's formula applied to $\left|\left(Y_{t}-Y_{t}^{\prime}\right)^{+}\right|^{2}$ yields that

$$
\begin{aligned}
\mid\left(Y_{t}-\right. & \left.Y_{t}^{\prime}\right)\left.^{+}\right|^{2} \\
= & 2 \int_{t}^{T}\left(Y_{t}-Y_{t}^{\prime}\right)^{+}\left(f\left(t, Y_{t}, \mathbb{E} Y_{t}, Z_{t}\right)\right. \\
& \left.-f^{\prime}\left(t, Y_{t}^{\prime}, \mathbb{E} Y_{t}^{\prime}, Z_{t}^{\prime}\right)\right) d t \\
& +2 \int_{t}^{T}\left(Y_{t}-Y_{t}^{\prime}\right)^{+}\left(d K_{t}-d K_{t}^{\prime}\right) \\
& -2 \int_{t}^{T}\left(Y_{t}-Y_{t}^{\prime}\right)^{+}\left(Z_{t}-Z_{t}^{\prime}\right) d B_{t} \\
& -\int_{t}^{T}\left(Y_{t}-Y_{t}^{\prime}\right)^{+} d L_{t}-\int_{t}^{T} \mathbf{1}_{\left\{Y_{t}>Y_{t}^{\prime}\right\}}\left|Z_{t}-Z_{t}^{\prime}\right|^{2} d t \\
\leq & 2 \int_{t}^{T}\left(Y_{t}-Y_{t}^{\prime}\right)^{+}\left(f\left(t, Y_{t}, \mathbb{E} Y_{t}, Z_{t}\right)\right. \\
& \quad+2 \int_{t}^{T}\left(Y_{t}-Y_{t}^{\prime}\right)^{+}\left(d K_{t}-d K_{t}^{\prime}\right) \\
& -2 \int_{t}^{T}\left(Y_{t}-Y_{t}^{\prime}\right)^{+}\left(Z_{t}-Z_{t}^{\prime}\right) d B_{t} \\
& -\int_{t}^{T} \mathbf{1}_{\left\{Y_{t}>Y_{t}^{\prime}\right\}}\left|Z_{t}-Z_{t}^{\prime}\right|^{2} d t .
\end{aligned}
$$

Taking expectations we find that

$$
\begin{aligned}
& \mathbb{E}\left|\left(Y_{t}-Y_{t}^{\prime}\right)^{+}\right|^{2} \\
& +\mathbb{E} \int_{t}^{T} \mathbf{1}_{\left\{Y_{s}>Y_{s}^{\prime}\right\}}\left|Z_{s}-Z_{s}^{\prime}\right|^{2} d s \\
& \leq 2 \mathbb{E} \int_{t}^{T}\left(Y_{s}-Y_{s}^{\prime}\right)^{+}\left(f\left(s, Y_{s}, \mathbb{E} Y_{s}, Z_{s}\right)\right. \\
& \left.-f^{\prime}\left(s, Y_{s}^{\prime}, \mathbb{E} Y_{s}^{\prime}, Z_{s}^{\prime}\right)\right) d s \\
& +2 \mathbb{E} \int_{t}^{T}\left(Y_{s}-Y_{s}^{\prime}\right)^{+}\left(d K_{s}-d K_{s}^{\prime}\right) .
\end{aligned}
$$

But on $\left\{Y_{t}>Y_{t}^{\prime}\right\}$ it holds that $Y_{t}>Y_{t}^{\prime} \geq S_{t}^{\prime} \geq S_{t}$, from which it follows that

$$
\int_{t}^{T}\left(Y_{s}-Y_{s}^{\prime}\right)^{+}\left(d K_{s}-d K_{s}^{\prime}\right)=-\int_{t}^{T}\left(Y_{s}-Y_{s}^{\prime}\right)^{+} d K_{s}^{\prime} \leq 0 .
$$

Hence

$$
\begin{aligned}
& \mathbb{E}\left|\left(Y_{t}-Y_{t}^{\prime}\right)^{+}\right|^{2} \\
& +\mathbb{E} \int_{t}^{T} \mathbf{1}_{\left\{Y_{s}>Y_{s}^{\prime}\right\}}\left|Z_{s}-Z_{s}^{\prime}\right|^{2} d s \\
& \leq 2 \mathbb{E} \int_{t}^{T}\left(Y_{s}-Y_{s}^{\prime}\right)^{+} \\
& \quad \times\left(f\left(s, Y_{s}, \mathbb{E} Y_{s}, Z_{s}\right)-f^{\prime}\left(s, Y_{s}^{\prime}, \mathbb{E} Y_{s}^{\prime}, Z_{s}^{\prime}\right)\right) d s \\
& =2 \mathbb{E} \int_{t}^{T}\left(Y_{s}-Y_{s}^{\prime}\right)^{+} \\
& \quad \times\left(f\left(s, Y_{s}, \mathbb{E} Y_{s}, Z_{s}\right)-f\left(s, Y_{s}^{\prime}, \mathbb{E} Y_{s}^{\prime}, Z_{s}^{\prime}\right)\right. \\
& \left.\quad+f\left(s, Y_{s}^{\prime}, \mathbb{E} Y_{s}^{\prime}, Z_{s}^{\prime}\right)-f^{\prime}\left(s, Y_{s}^{\prime}, \mathbb{E} Y_{s}^{\prime}, Z_{s}^{\prime}\right)\right) d s \\
& \leq 2 \mathbb{E} \int_{t}^{T}\left(Y_{s}-Y_{s}^{\prime}\right)^{+} \\
& \quad \times\left(f\left(s, Y_{s}, \mathbb{E} Y_{s}, Z_{s}\right)-f\left(s, Y_{s}^{\prime}, \mathbb{E} Y_{s}^{\prime}, Z_{s}^{\prime}\right)\right) d s
\end{aligned}
$$

by assumption. Without loss of generality we may assume that $f$ is the generator which is nondecreasing in the third argument so that by the Lipschitz property of $f$ we get

$$
\begin{aligned}
& \mathbb{E}\left|\left(Y_{t}-Y_{t}^{\prime}\right)^{+}\right|^{2} \\
& +\mathbb{E} \int_{t}^{T} \mathbf{1}_{\left\{Y_{s}>Y_{s}^{\prime}\right\}}\left|Z_{s}-Z_{s}^{\prime}\right|^{2} d s \\
& \leq 2 K \mathbb{E} \int_{t}^{T}\left(Y_{s}-Y_{s}^{\prime}\right)^{+} \\
& \quad \times\left(\left|Y_{s}-Y_{s}^{\prime}\right|+\left(\mathbb{E} Y_{s}-\mathbb{E} Y_{s}^{\prime}\right)^{+}+\left|Z_{s}-Z_{s}^{\prime}\right|\right) d s \\
& \leq 2 K \mathbb{E} \int_{t}^{T}\left(Y_{s}-Y_{s}^{\prime}\right)^{+}\left(\left|Y_{s}-Y_{s}^{\prime}\right|+\left|Z_{s}-Z_{s}^{\prime}\right|\right) d s \\
& \quad+2 K \mathbb{E} \int_{t}^{T}\left(Y_{s}-Y_{s}^{\prime}\right)^{+} \mathbb{E}\left[\left(Y_{s}-Y_{s}^{\prime}\right)^{+}\right] d s,
\end{aligned}
$$

where the last inequality follows from Jensen's inequality. For the first integral we have

$$
\begin{aligned}
2 K \mathbb{E} \int_{t}^{T}\left(Y_{s}-Y_{s}^{\prime}\right)^{+}\left(\left|Y_{s}-Y_{s}^{\prime}\right|+\left|Z_{s}-Z_{s}^{\prime}\right|\right) d s \\
=2 K \mathbb{E} \int_{t}^{T}\left|\left(Y_{s}-Y_{s}^{\prime}\right)^{+}\right|^{2} d s \\
\quad+\mathbb{E} \int_{t}^{T} 2 K\left(Y_{s}-Y_{s}^{\prime}\right)^{+} \mathbf{1}_{\left\{Y_{s}>Y_{s}^{\prime}\right\}}\left|Z_{s}-Z_{s}^{\prime}\right| d s
\end{aligned}
$$




$$
\begin{aligned}
\leq & 2 K \mathbb{E} \int_{t}^{T}\left|\left(Y_{s}-Y_{s}^{\prime}\right)^{+}\right|^{2} d s \\
& +2 K^{2} \mathbb{E} \int_{t}^{T}\left|\left(Y_{s}-Y_{s}^{\prime}\right)^{+}\right|^{2} d s \\
& +\frac{1}{2} \mathbb{E} \int_{t}^{T} \mathbf{1}_{\left\{Y_{s}>Y_{s}^{\prime}\right\}}\left|Z_{s}-Z_{s}^{\prime}\right|^{2} d s \\
= & \left(2 K+2 K^{2}\right) \mathbb{E} \int_{t}^{T}\left|\left(Y_{s}-Y_{s}^{\prime}\right)^{+}\right|^{2} d s \\
& +\frac{1}{2} \mathbb{E} \int_{t}^{T} \mathbf{1}_{\left\{Y_{s}>Y_{s}^{\prime}\right\}}\left|Z_{s}-Z_{s}^{\prime}\right|^{2} d s .
\end{aligned}
$$

Inserting into the above and canceling out terms yield that

$$
\begin{aligned}
\mathbb{E}\left|\left(Y_{t}-Y_{t}^{\prime}\right)^{+}\right|^{2} & \frac{1}{2} \mathbb{E} \int_{t}^{T} \mathbf{1}_{\left\{Y_{s}>Y_{s}^{\prime}\right\}}\left|Z_{s}-Z_{s}^{\prime}\right|^{2} d s \\
\leq & \left(2 K+2 K^{2}\right) \mathbb{E} \int_{t}^{T}\left|\left(Y_{s}-Y_{s}^{\prime}\right)^{+}\right|^{2} d s \\
& +2 K \mathbb{E} \int_{t}^{T}\left(Y_{s}-Y_{s}^{\prime}\right)^{+} \mathbb{E}\left[\left(Y_{s}-Y_{s}^{\prime}\right)^{+}\right] d s \\
= & \left(2 K+2 K^{2}\right) \mathbb{E} \int_{t}^{T}\left|\left(Y_{s}-Y_{s}^{\prime}\right)^{+}\right|^{2} d s \\
& +2 K \int_{t}^{T} \mathbb{E}\left[\left(Y_{s}-Y_{s}^{\prime}\right)^{+}\right]^{2} d s \\
\leq & \left(2 K+2 K^{2}\right) \mathbb{E} \int_{t}^{T}\left|\left(Y_{s}-Y_{s}^{\prime}\right)^{+}\right|^{2} d s \\
= & \left(4 K+2 K^{2}\right) \mathbb{E} \int_{t}^{T}\left|\left(Y_{s}-Y_{s}^{\prime}\right)^{+}\right|^{2} d s . \\
& +2 K \int_{t}^{T} \mathbb{E}\left[\left|\left(Y_{s}-Y_{s}^{\prime}\right)^{+}\right|^{2}\right] d s
\end{aligned}
$$

Hence, it holds that

$$
\mathbb{E}\left|\left(Y_{t}-Y_{t}^{\prime}\right)^{+}\right|^{2} \leq\left(4 K+K^{2}\right) \mathbb{E} \int_{t}^{T}\left|\left(Y_{s}-Y_{s}^{\prime}\right)^{+}\right|^{2} d s
$$

Using Gronwall's inequality yields that

$$
\mathbb{E}\left|\left(Y_{t}-Y_{t}^{\prime}\right)^{+}\right|^{2}=0
$$

and hence $Y_{t} \leq Y_{t}^{\prime} \mathbb{P}$-a.s.

\section{Proof of Proposition 5}

Consider the penalized equations of the MF-RBSDE for $n \geq$ 1 :

$$
\begin{aligned}
Y_{t}^{n}= & \xi+\int_{t}^{T} f\left(s, Y_{s}^{n}, \mathbb{E} Y_{s}^{n}, Z_{s}^{n}\right) d s \\
& +n \int_{t}^{T}\left(Y_{s}^{n}-S_{s}\right)^{-} d s-\int_{t}^{T} Z_{s}^{n} d B_{s}, \\
Y_{t}^{\prime, n}= & \xi^{\prime}+\int_{t}^{T} f^{\prime}\left(s, Y_{s}^{\prime, n}, \mathbb{E} Y_{s}^{\prime, n}, Z_{s}^{\prime, n}\right) d s \\
& +n \int_{t}^{T}\left(Y_{s}^{\prime, n}-S_{s}\right)^{-} d s-\int_{t}^{T} Z_{s}^{\prime, n} d B_{s} .
\end{aligned}
$$

Letting

$$
\begin{aligned}
f^{n}\left(t, y_{1}, y_{2}, z\right) & :=f\left(t, y_{1}, y_{2}, z\right)+n\left(y_{2}-z\right)^{-} \\
f^{\prime, n}\left(t, y_{1}, y_{2}, z\right) & :=f^{\prime}\left(t, y_{1}, y_{2}, z\right)+n\left(y_{2}-z\right)^{-}
\end{aligned}
$$

we have two sequences of unreflected continuous MF-BSDEs with generators $f^{n}$ and $f^{\prime, n}$, where

$$
f^{n}\left(t, y_{1}, y_{2}, z\right) \leq f^{\prime, n}\left(t, y_{1}, y_{2}, z\right)
$$

Following the steps in the proof of Proposition 4 we see that $Y_{t}^{n} \leq Y_{t}^{1, n}$ a.s. Taking the limit $n \rightarrow \infty$ we obtain the result.

\section{Conflict of Interests}

The authors declare that there is no conflict of interests regarding the publication of this paper.

\section{Acknowledgment}

The financial support from The Swedish Export Credit Corporation (SEK) is gratefully acknowledged.

\section{References}

[1] B. Djehiche and A. A. Hamdi, "Full balance sheet two-modes optimal switching problem," Preprint, 2011.

[2] N. El Karoui, C. Kapoudjian, E. Pardoux, S. Peng, and M. C. Quenez, "Reflected solutions of backward SDE's, and related obstacle problems for PDE's," The Annals of Probability, vol. 25, no. 2, pp. 702-737, 1997.

[3] V. S. Borkar and K. S. Kumar, "McKean-Vlasov limit in portfolio optimization," Stochastic Analysis and Applications, vol. 28, no. 5, pp. 884-906, 2010.

[4] J. M. Lasry and P. L. Lions, "Mean field games," Japanese Journal of Mathematics, vol. 2, no. 1, pp. 229-260, 2007.

[5] R. Buckdahn, B. Djehiche, J. Li, and S. Peng, "Mean-field backward stochastic differential equations: a limit approach," The Annals of Probability, vol. 37, no. 4, pp. 1524-1565, 2009.

[6] R. Buckdahn, J. Li, and S. Peng, "Mean-field backward stochastic differential equations and related partial differential equations," Stochastic Processes and Their Applications, vol. 119, no. 10, pp. 3133-3154, 2009. 
[7] J. Li, "Reflected mean-field backward stochastic differential equations. Approximation and associated nonlinear PDEs," Journal of Mathematical Analysis and Applications, vol. 413, no. 1, pp. 47-68, 2014.

[8] Z. Li and J. Luo, "Mean-field reflected backward stochastic differential equations," Statistics \& Probability Letters, vol. 82, no. 11, pp. 1961-1968, 2012.

[9] J. Cvitanić and I. Karatzas, "Backward stochastic differential equations with reflection and Dynkin games," The Annals of Probability, vol. 24, no. 4, pp. 2024-2056, 1996.

[10] S. Hamadène, "Reflected BSDE's with discontinuous barrier and application," Stochastics and Stochastics Reports, vol. 74, no. 3-4, pp. 571-596, 2002.

[11] I. Karatzas and S. E. Shreve, Methods of Mathematical Finance, vol. 39, Springer, New York, NY, USA, 1998.

[12] C. Dellacherie and P. Meyer, Probabilities and Potential, chapter 1-4, Hermann, Paris, France, 1975.

[13] S. Peng, "Monotonic limit theorem of BSDE and nonlinear decomposition theorem of Doob-Meyer's type," Probability Theory and Related Fields, vol. 113, no. 4, pp. 473-499, 1999. 


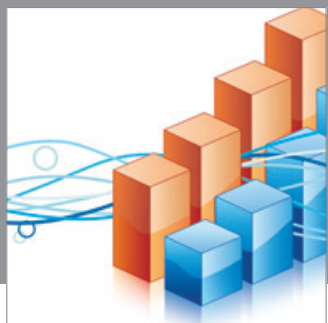

Advances in

Operations Research

mansans

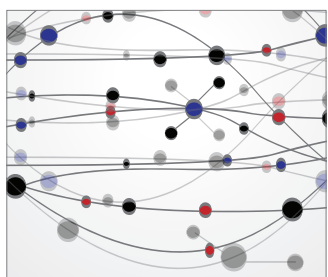

The Scientific World Journal
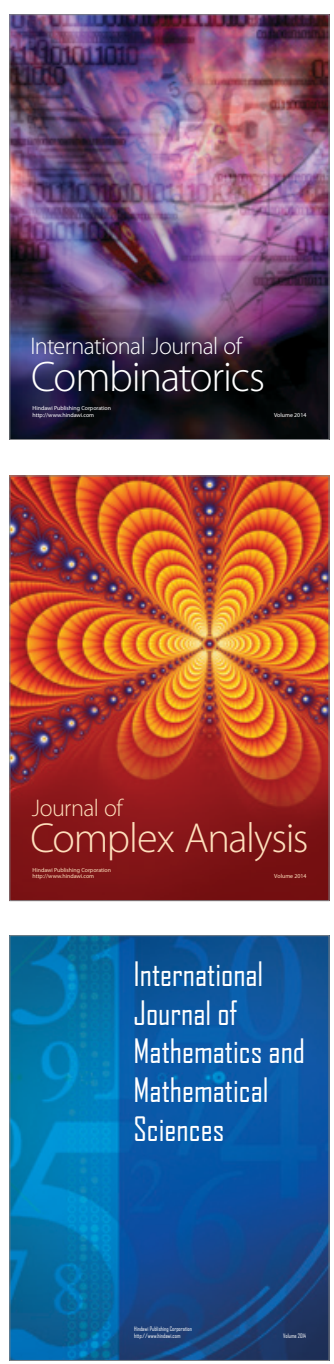
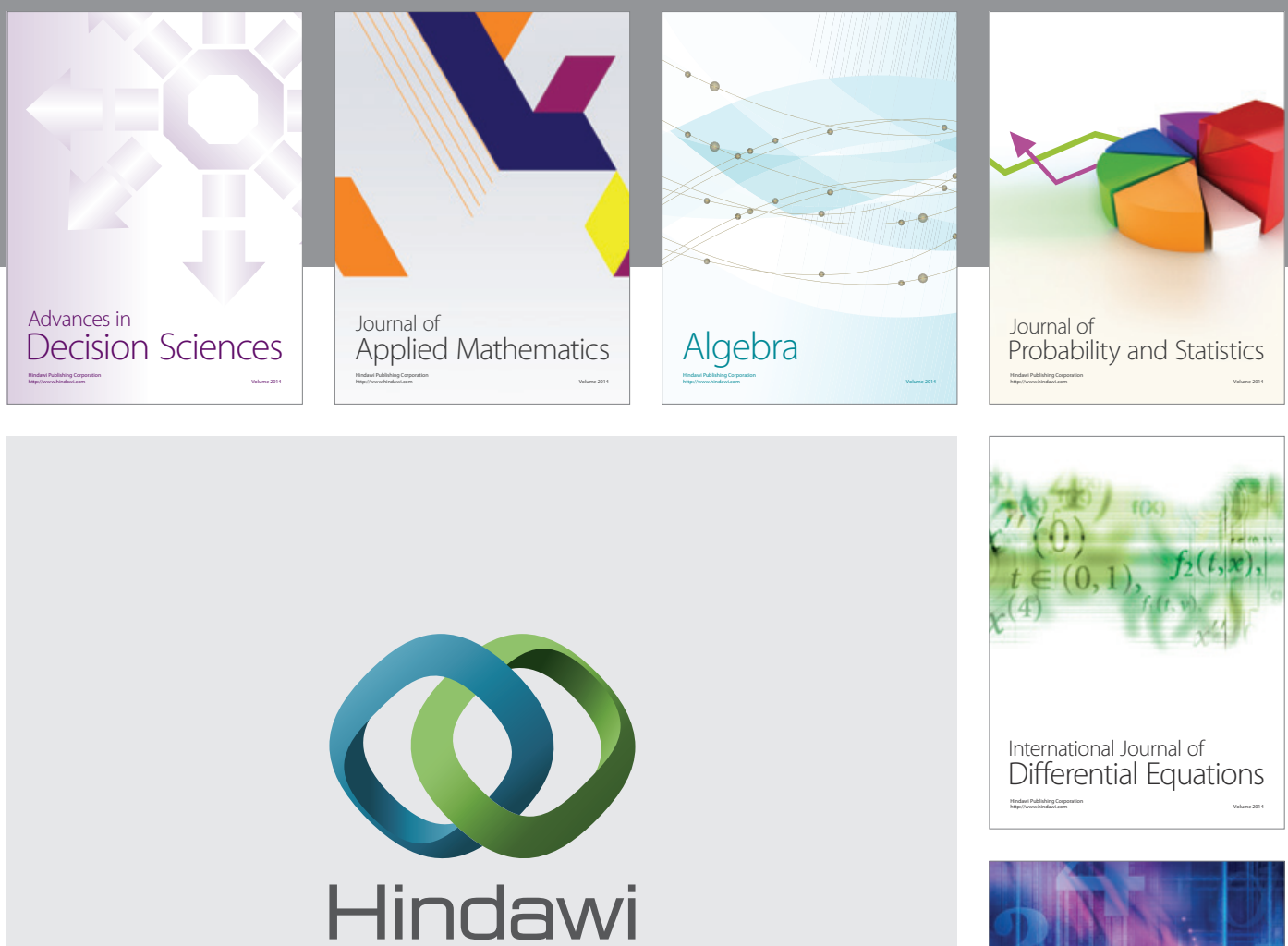

Submit your manuscripts at http://www.hindawi.com
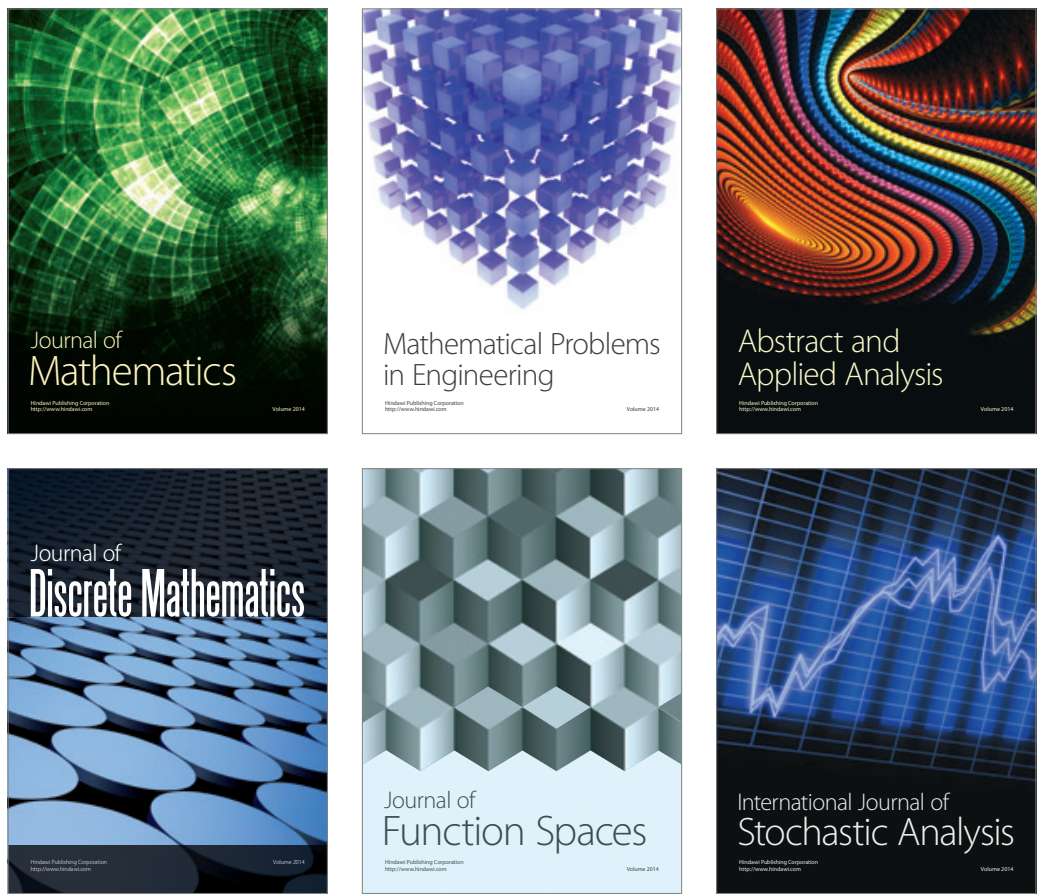

Journal of

Function Spaces

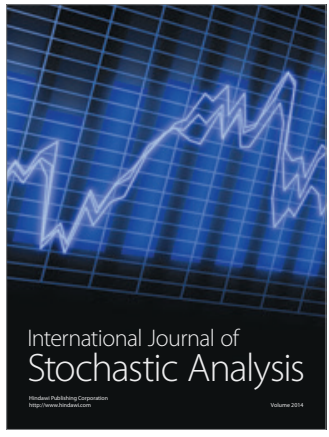

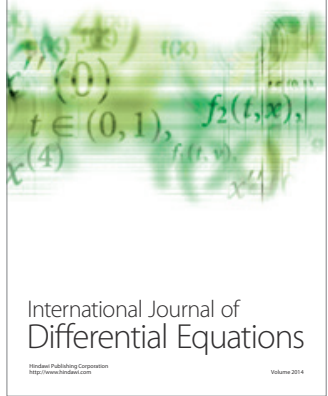
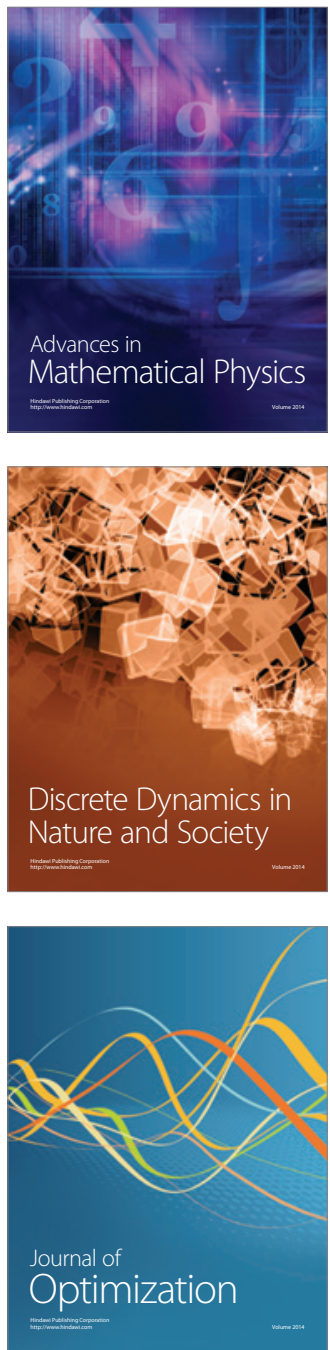